\title{
Engrailed Alters the Specificity of Synaptic Connections of Drosophila Auditory Neurons with the Giant Fiber
}

\author{
Adeline Pézier, Sami H. Jezzini, Bruno Marie, and Jonathan M. Blagburn \\ Institute of Neurobiology, University of Puerto Rico, San Juan, Puerto Rico 00901
}

We show that a subset of sound-detecting Johnston's Organ neurons (JONs) in Drosophila melanogaster, which express the transcription factors Engrailed (En) and Invected (Inv), form mixed electrical and chemical synaptic inputs onto the giant fiber (GF) dendrite. These synaptic connections are detected by trans-synaptic Neurobiotin (NB) transfer and by colocalization of Bruchpilot-short puncta. We then show that misexpressing En postmitotically in a second subset of sound-responsive JONs causes them to form ectopic electrical and chemical synapses with the GF, in turn causing that postsynaptic neuron to redistribute its dendritic branches into the vicinity of these afferents. We also introduce a simple electrophysiological recording paradigm for quantifying the presynaptic and postsynaptic electrical activity at this synapse, by measuring the extracellular sound-evoked potentials (SEPs) from the antennal nerve while monitoring the likelihood of the GF firing an action potential in response to simultaneous subthreshold sound and voltage stimuli. Ectopic presynaptic expression of En strengthens the synaptic connection, consistent with there being more synaptic contacts formed. Finally, RNAimediated knockdown of En and Inv in postmitotic neurons reduces SEP amplitude but also reduces synaptic strength at the JON-GF synapse. Overall, these results suggest that En and Inv in JONs regulate both neuronal excitability and synaptic connectivity.

Key words: Drosophila; gap junctions; giant fiber system; mechanosensory; synapses; transcription factor

\section{Introduction}

The transcription factor Engrailed (En) was first identified in Drosophila, in which it was shown to determine the posterior identity of body segments (Morata and Lawrence, 1975; Kornberg, 1981). It was subsequently found to be present in all animals, including humans (Gibert, 2002), and its most conserved role seems to be in neuronal development.

In vertebrates, En is required for patterning the cerebellum (Baader et al., 1999; Sillitoe et al., 2010) and for guiding retinal axons in the tectum (Friedman and O'Leary, 1996; Itasaki and Nakamura, 1996; Logan et al., 1996; Shigetani et al., 1997). En regulates the development of some spinal cord interneurons (Wenner et al., 2000; Simon et al., 2001) and affects the survival of

Received May 13, 2014; revised July 8, 2014; accepted July 20, 2014.

Author contributions: A.P., S.H.J., and J.M.B. designed research; A.P., S.H.J., and J.M.B. performed research; B.M. contributed unpublished reagents/analytic tools; A.P., S.H.J., and J.M.B. analyzed data; A.P., S.H.J., B.M., and J.M.B. wrote the paper.

This work was supported by National Institute of Neurological Disorders and Stroke Grants SC1 NS081726 (J.M.B.) and SC2 NS077924 (B.M.). The Institute of Neurobiology confocal microscope was funded by National Science Foundation Grant DBI 0115825, Department of Defense Grant 52680-RT-ISP, and National Institute on Minority Health and Health Disparities (NIMHD) Grant 8G12-MD007600 (Research Centers in Minority Institutions). The Institute of Neurobiology Drosophila resource center was supported by NIMHD Grant G12MD007600 (Research Centers in Minority Institutions). The monoclonal antibodies developed by Dr. Corey Goodman and Dr. E. Buchner were obtained from the Developmental Studies Hybridoma Bank developed under the auspices of the National Institute of Child Health and Human Development and maintained by the Department of Biological Sciences, University of lowa (lowa City, IA). We also acknowledge the Vienna Drosophila RNAi Center for the supply of fly lines. This work was made possible in part by software funded by National Institutes of Health Grant R01-GM098151-01: Fluorender: An Imaging Tool for Visualization and Analysis of Confocal Data as Applied to Zebrafish Research.

The authors declare no competing financial interests.

Correspondence should be addressed to Jonathan M. Blagburn, Institute of Neurobiology, 201 Boulevard del Valle, San Juan, PR 00901. E-mail: jonathan.blagburn@upr.edu.

DOI:10.1523/JNEUROSCI.1939-14.2014

Copyright $\odot 2014$ the authors $\quad 0270-6474 / 14 / 3411691-14 \$ 15.00 / 0$ dopaminergic midbrain neurons (Simon et al., 2001; Sgadò et al., 2006). In Drosophila and grasshopper CNS, En controls neuron/ glia fate decisions, neuronal identity, and axon pathfinding (Condron et al., 1994; Lundell et al., 1996; Joly et al., 2007), whereas in cockroach mechanosensory neurons we showed that it also controls axon guidance, synaptic target recognition, and, as a result, escape behavior (Marie et al., 2000, 2002; Marie and Blagburn, 2003; Blagburn and Bacon, 2004; Booth et al., 2009).

En expression also persists beyond development in the PNS and CNS of adult Drosophila (Blagburn, 2008). It is restricted to subsets of neurons in which its roles are just beginning to be explored. In combination with other transcription factors, it is necessary for specifying olfactory sensillum identity and Or odorant receptor gene expression in the third antennal segment (Jafari et al., 2012; Song et al., 2012). We described recently the pattern of En expression in a posterior subset of the mechanosensory neurons belonging to the Johnston's Organ (JO), located in the second antennal segment (Pézier and Blagburn, 2013; Fig. 1).

This large chordotonal organ contains $\sim 480$ sensory neurons (Kamikouchi et al., 2006; Boekhoff-Falk and Eberl, 2014). Some of these JO neurons (JONs) detect sound (JO-A and JO-B subtypes: Fig. 1), whereas another subpopulation responds to gravity and wind (JO-C and JO-E; Kamikouchi et al., 2009; Sun et al., 2009; Yorozu et al., 2009; Lehnert et al., 2013). We found that En-expressing JONs respond to air movements from both the front and back of the animal and that they respond to 100-400 Hz tones (Pézier and Blagburn, 2013).

Cobalt fills showed that the giant fiber neuron (GF) receives gap junctional synaptic input from JONs (Strausfeld and Bassemir, 1983; Bacon and Strausfeld, 1986). Recently, these electrical synaptic inputs have been recorded directly from the GF cell 
A
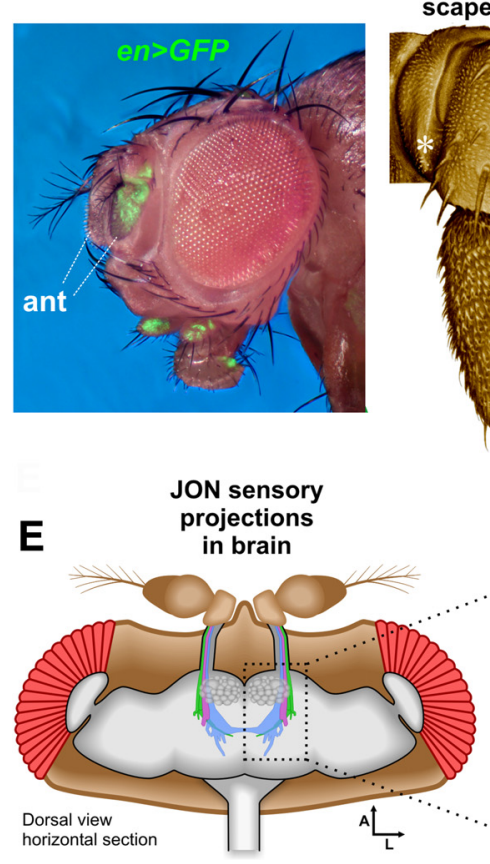

B

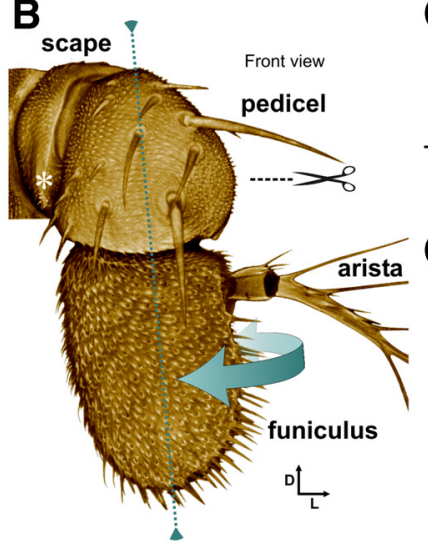

C1 JoN subgroups D1

D1 en>GFP

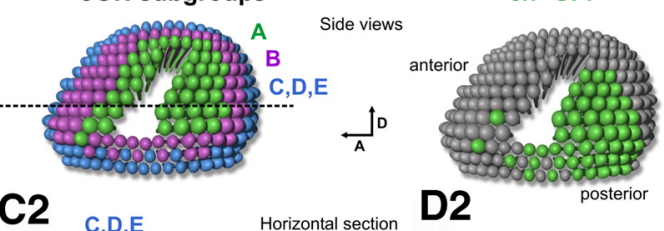

C2

Horizontal section

F

FoN projection zones

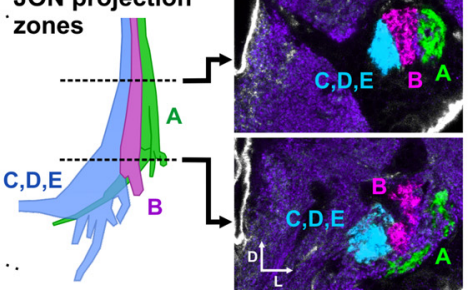

Frontal sections

G

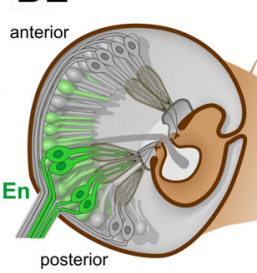

an $B, D, E$

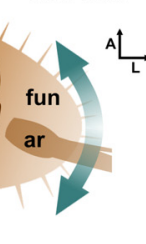

posterior

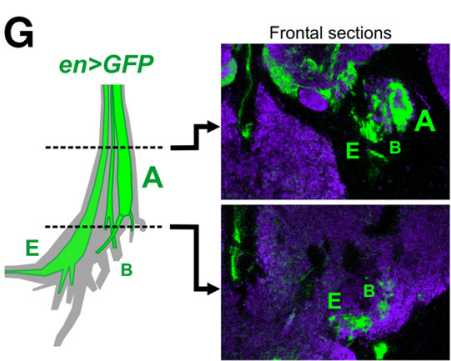

Figure 1. En is expressed in a subset of JONs that contact the GF. $A$, Digitally superimposed epifluorescence and reflected light micrographs of the head of an animal expressing en-driven CD8::GFP, showing strong GFP fluorescence in the antennae (ant). $\boldsymbol{B}, 3 \mathrm{D}$ front view of left antenna. Rotational axis of funiculus shown in green. Cut mark indicates transverse sections shown in $\mathbf{C}$ and $\mathbf{D 2}$. The white asterisk indicates recording electrode insertion site. C, Anatomical subtypes of JONs. C1,3D diagram of the bowl-shaped array of JON cell bodies in the right antenna, viewed from the medial side, divided into groups by axonal projection type: $\mathrm{A}$ (green), B (magenta), and $\mathrm{C}-\mathrm{E}$ (blue). Line indicates position of sections in $\mathbf{C}$ and $\mathbf{D 2}$. Anterior is to the left. $\mathbf{C 2}$, Diagram of a transverse section through the pedicel and J0, showing the relative positions of the different JON groups. Curved arrows indicate the movement of the funiculus (fun) and arista (ar). Two JONs are grouped in a single scolopidium, together with scolopale cells (gray ovals). Their dendrites are stretched by movements of the funiculus. $D$, JONs that express en-driven (D8::GFP (green), showing their 3D arrangement (D1) and their position in a horizontal section of the pedicel (D2). E, Diagram of a horizontal section through the head, showing the JON sensory projections in the brain. $\boldsymbol{F}$, Detailed diagram of the different projection zones, showing A (green), B (magenta), and C $-E$ (blue) JON groups. To the right are frontal confocal sections taken from the positions indicated, false colored to show the different arbor types, with neuropil staining shown in dark purple. G, Diagram of the projections of the en-expressing JONs (green), with most axons in the $A$ and E groups and a few in the B group. To the right are frontal confocal sections showing en-driven GFP fluorescence. Throughout, arrows indicate dorsal (D), lateral (L), and anterior (A) directions.

body in response to sound and air movements (Tootoonian et al., 2012; Lehnert et al., 2013; Mu et al., 2014), and it appears that a dendrite of GF is inserted directly in the region of JO-A afferent projections (Lehnert et al., 2013). In this study, we find that Enexpressing JONs are the ones that form these synapses and show anatomically and electrophysiologically that perturbation of En expression in the JONs alters their synaptic connections with the GF.

\section{Materials and Methods}

Flies. Drosophila melanogaster were reared on cornmeal media and raised at $25^{\circ} \mathrm{C}$. In some cases, to increase GAL4 activity, flies were transferred to $30^{\circ} \mathrm{C}$ or, to decrease it, to $18^{\circ} \mathrm{C}$ (Duffy, 2002). Flies of the following genotypes were obtained from the Bloomington Stock Center: en-GAL4 el6E (30564), JO15-GAL4 (6753), UAS-mCD8::GFP (5137), $w^{*} ; P\{E P\}-$ inv ${ }^{G E 10665}$ (26891), UAS-Dcr-2 (24650), UAS-enRNAi (26752: $y^{1} v^{1}$; P\{TRiP.JF02316\}attP2 or 33715: $y^{1} s c^{*} v^{1}$;P\{TRiP.HMS00595\}attP2), UAS-brpRNAi (25891), and $w^{1118} P\{G a w B\} B x^{M S 1096}$ (8860). UAS-invR$N A i$ (49952) was obtained from the Vienna Drosophila RNAi Center (Dietzl et al., 2007). Other lines used were UAS-en [Miki Fujioka, Philadephia, PA (Guillén et al., 1995)], UAS-brp-sh-strawberry [Stephan Sigrist, Berlin, Germany (Christiansen et al., 2011)], shakB ${ }^{2}$ [Tanja Godenschwege, Boca Raton, FL (Homyk et al., 1980)], UAS-TeTxLC [Tanja Godenschwege (Sweeney et al., 1995)], and peb-GAL4 and pebGAL4, mCD8::GFP [Liqun Luo, Stanford, CA (Sweeney et al., 2007)]. The following fly lines were constructed in the laboratory: (1) UAS-mCD8::GFP/CyO, Kr-GFP;JO15-GAL4/TM6B, Tb ${ }^{1}$; (2) peb-GAL4; UAS-Dcr-2/CyO, Kr-GFP; (3) peb-GAL4, mCD8::GFP;UAS-Dcr-2/CyO, KrGFP; (4) UAS-Dcr-2/CyO, Kr-GFP;JO15-GAL4/TM6B, Tb ${ }^{1}$; (5) UAS-brp-sh-
strawberry/CyO, Kr-GFP;UAS-en/TM6B, $\mathrm{Tb}^{1}$; (6) UAS-invRNAi/CyO, KrGFP; UAS-enRNAi/TM6B, $\mathrm{Tb}^{l}$; and (7) UAS-TeTxLC/CyO, Kr-GFP;UASen/TM6B, $T b^{1}$.

Dye coupling and immunohistochemistry. Adults of either sex were used up to $10 \mathrm{~d}$ after eclosion. The animals were anesthetized by cooling. Dissections were performed under a dissecting microscope as described previously (Boerner and Godenschwege, 2011). After the removal of the wings, legs, and proboscis, flies were pinned dorsal side up in a Sylgard (Dow Corning)-coated dish using one pin through the posterior of the abdomen and a pin on either side of the neck. After flooding the dish with Drosophila saline (in mM: $135 \mathrm{NaCl}, 5 \mathrm{KCl}, 4 \mathrm{MgCl}_{2}, 2 \mathrm{CaCl}_{2}, 5 \mathrm{TES}$, and 36 sucrose, pH 7.2) (Marley and Baines, 2011), a dorsal incision was made along the midline of the abdomen and thorax using fine scissors. The thorax was then pinned open, and overlying viscera were removed to expose the entire length of the central nerve cord, including the cervical connective. The preparation was then moved to a Zeiss Axio Examiner D1 compound microscope with which the GF was visualized in the cervical connective under differential interference contrast optics using a $40 \times$ water-immersion lens.

Intracellular dye was prepared in deionized water as a mixture of 3\% NB (Vector Laboratories) and 2\% Lucifer yellow CH, lithium salt (LY) (Molecular Probes). One millimeter thin-walled glass electrodes were backfilled with $0.6 \mu \mathrm{l}$ of dye mixture, followed by $150 \mathrm{~mm} \mathrm{KCl}$. Injection electrodes typically had resistances of $45-60 \mathrm{M} \Omega$ when filled with these solutions. After impalement of the GF, dye was iontophoretically injected for up to $20 \mathrm{~min}$. We used a continuous train of alternating square pulses of positive and negative current with amplitudes of $\pm 1-2 \mathrm{nA}$ and a duration of $1 \mathrm{~s}$ each ( $2 \mathrm{~s}$ cycle period). The pulses were delivered through an AxoClamp 2B amplifier (Molecular Devices) and were generated by a 
Master 8 stimulus generator (A.M.P.I.). Signals were displayed on a computer using a Digidata 1440A digitizer and Clampex 10 software (Molecular Devices). The progress and stability of the injection current were continuously monitored, and the quality of the LY injection was assessed visually by florescence.

Immediately after injection, preparations were fixed in $4 \%$ paraformaldehyde in PBS for $30 \mathrm{~min}$ at $4^{\circ} \mathrm{C}$. After fixation, preparations were rinsed in several changes of PBS and further dissected to remove the brain. After removal, brains or pupal antennae were processed for antibody labeling, cleared, and mounted as described previously (Blagburn, 2008; Pézier and Blagburn, 2013). The nc82 [anti-Bruchpilot (Brp); Wagh et al., 2006] and the 4D9 [anti-En and Invected (Inv); Patel et al., 1989] antibodies were obtained from the Developmental Studies Hybridoma Bank and used at a dilution of 1:20. Anti-En rabbit polyclonal antibody (D-300; Santa Cruz Biotechnology) was used at 1:200. Anti-LY (Invitrogen) was used at a dilution of 1:1000. Goat anti-mouse and antirabbit secondary antibodies labeled with Alexa Fluor-488, Alexa Fluor555, or Pacific Blue (Invitrogen) were applied at a dilution of 1:400. Avidin-conjugated Pacific Blue (Invitrogen) was added at 1:1000 during incubation in primary and secondary antibody. Preparations were examined with a Zeiss Pascal laser-scanning confocal microscope, and images were acquired at 8-bit resolution.

Image processing and analysis. For most figures, image stacks were imported into NIH ImageJ (Schneider et al., 2012) or Fiji (Schindelin et al., 2012), in which they were adjusted for optimal contrast. 3D projections of $z$-series were made using FluoRender (Wan et al., 2012) and were imported into Adobe Photoshop (Adobe Systems) for construction of figures. For clarity, before making some projections (Fig. $2 G-I$ ), non-JO neuronal GFP fluorescence was digitally masked from the stack. Dendritic branches were traced, and their lengths were measured using the Simple Neurite Tracer plugin of Fiji. Other figures were composed using Blender (www.Blender.org), CorelDraw (Corel), and Photoshop, in which they were checked for color-blind accessibility using the Vischeck plugin (www.vischeck.com).

For image quantification, data from control and experimental preparations were acquired and processed in the same way. For Figure 3, images were thresholded manually in NIH ImageJ and then binarized, and the area of signal was measured for 30 of the $1 \mu \mathrm{m}$ slices, beginning with the most anterior one in which the GF dendrite appeared. For Figure 4, the Brp-short (Brp-sh) channel (red) was binarized without any preprocessing using the RenyiEntropy method in the autothreshold menu of NIH ImageJ, with the "ignore white" parameter set to exclude saturated pixels from the calculation. Only the brightest areas of labeling were retained in the neuropil, resulting in a somewhat conservative measure. For the LY channel, automatic contrast enhancement and background subtraction were done before autothresholding using the RenyiEntropy method. This produced a slight overestimation of dendritic diameters but retained more of the fine processes. Most of the NB images were simply autothresholded using the RenyiEntropy method without any preprocessing. In some preparations with lower labeling intensities, a bleach correction function was used before thresholding to compensate for the declining signal intensity with stack depth. A region of interest (ROI) was defined by the extent of Brp-sh labeling in the antennal projection, with the $z$ dimension beginning two or three sections before the anteriormost GF dendrite and continuing posteriorly to the end of the JON arborizations. This range typically covered between 60 and 70 $\mu \mathrm{m}$. The channels were split for quantification of signal areas. The GF was defined as the overlap between the LY and NB channels. Presumptive synaptic zones were defined as overlap between the GF and Brp-sh channels (Fig. $4 B, D$, middle). Large cell-body agglomerations (Fig. $4 A, C$, asterisks) were manually removed from the Brp-sh ROI before quantitation.

Electrophysiology. Recordings were performed 3-10 d after eclosion in females. Preparation of flies, recording of the sound-evoked potential (SEP) from the antennal nerve, and sound delivery were performed as described previously (Pézier and Blagburn, 2013). Recordings from the GF system were performed simultaneously with the antennal nerve recording. The dorsal longitudinal flight muscle (DLM) and tergotrochanteral jump muscle (TTM) of the opposite side were recorded using glass electrodes filled with a 50:50 mix of $1 \mathrm{M} \mathrm{KCl}$ and $1 \mathrm{~m} \mathrm{~K}$-acetate. Both signals from each muscle were amplified $10 \times$, one with a Neuroprobe 1600 amplifier (A-M Systems) and the other one with a Intra 767 Electrometer (WPI). Signals were amplified another $10 \times$ and filtered at 30 $\mathrm{kHz}$ with a Brownlee Precision 210A amplifier, digitized with a Digidata $1320 \mathrm{~A}$, and acquired and sampled at $50 \mathrm{kHz}$ with pClamp. A tungsten electrode was placed into the abdomen to ground the preparation. In this study, we exclusively use the long-latency (3-5 ms) muscle response, obtained when a low intensity voltage is applied across the eyes (Tanouye and Wyman, 1980) and attributable to the activation of the GF through the polysynaptic visual pathway. Voltage stimuli were generated with a tungsten electrode inserted shallowly into each eye and connected to a S48 stimulator and SIU5 Isolation Unit (Grass Technologies) and were of $0.6 \mathrm{~ms}$ duration and given at $0.3 \mathrm{~Hz}$ to avoid habituation (Engel and $\mathrm{Wu}$, 1996). The voltage stimulus intensity necessary to reach the threshold for eliciting long-latency responses was predetermined and then adjusted for the experiment so as to be just subthreshold, so that muscle spikes, indicative of a GF action potential, occurred in only $\leq 1$ in 10 trials in the absence of sound, presumably because of small fluctuations in the amplitude of the visual input. If these criteria for adjusting the subthreshold stimulus voltage were consistently followed, the results obtained were remarkably similar across preparations.

The auditory stimulus consisted of $200 \mathrm{~Hz}$ sine waves of $100 \mathrm{~ms}$ duration. For each presynaptic SEP investigated, the delay of the voltage stimulus was adjusted so that the GF would receive the auditory and visual inputs in synchrony. The experiment then consisted of testing a series of sound levels varying from 78 to $98 \mathrm{~dB}$ for each SEP investigated, with, for each sound level, 10 trials in the presence of sound alternating with 10 trials in the absence of sound, all trials simultaneously applied with the subthreshold voltage stimulation. The timing of the voltage stimulus relative to the SEPs was critical. It was found empirically that optimal responses were achieved if the voltage stimulus onset preceded the peak of the SEP of interest by $\sim 3.5 \mathrm{~ms}$, so that the small transient of unknown origin that invariably precedes the TTM action potential (see Fig. 6D) coincided with the end of the SEP (see Fig. $6 B, D$, dashed arrows).

In total, between 50 and 100 sound trials were generated per SEP investigated per animal. SEP amplitudes were measured using Clampfit (Molecular Devices) and binned for each SEP investigated for each animal. The probability of the GF spiking was calculated as the number of trials with GF firing divided by the total number of trials for that amplitude bin. The probability of the GF spiking per amplitude bin was then averaged across animals of the same genotype. The GF was scored as spiking when both the TTM and the DLM fired with the appropriate latency.

Statistics. Data are presented as mean \pm SEM. $n$ is the number of animals. The normality of the distribution of the datasets was first determined using tests in PAST software (Hammer et al., 2001). All statistical tests were then performed using KyPlot (KyensLab). To identify significant differences between means of control versus experimental groups, normally distributed data were compared using Student's $t$ test, whereas non-normally distributed data were compared using a Mann-Whitney $U$ test. Significance between multiple groups was assessed with a one-way ANOVA. In figures, ${ }^{*} p \leq 0.05,{ }^{* *} p \leq 0.01,{ }^{* * *} p \leq 0.001$. Specific $p$ values are given in Results.

\section{Results}

\section{The GF is NB-coupled to En-expressing JONs}

We used an en-GAL4 line to drive CD8::GFP (GFP targeted to the membrane) expression in en-expressing neurons; as described previously, this appears to mirror faithfully the expression pattern of En protein (Blagburn, 2008; Pézier and Blagburn, 2013). Within the brain, En is expressed in a range of sensory and other neurons, as well as axons of the JONs. In the JO, en-expressing JONs lie mainly in the posterior region (Fig. 1D; Pézier and Blag- 

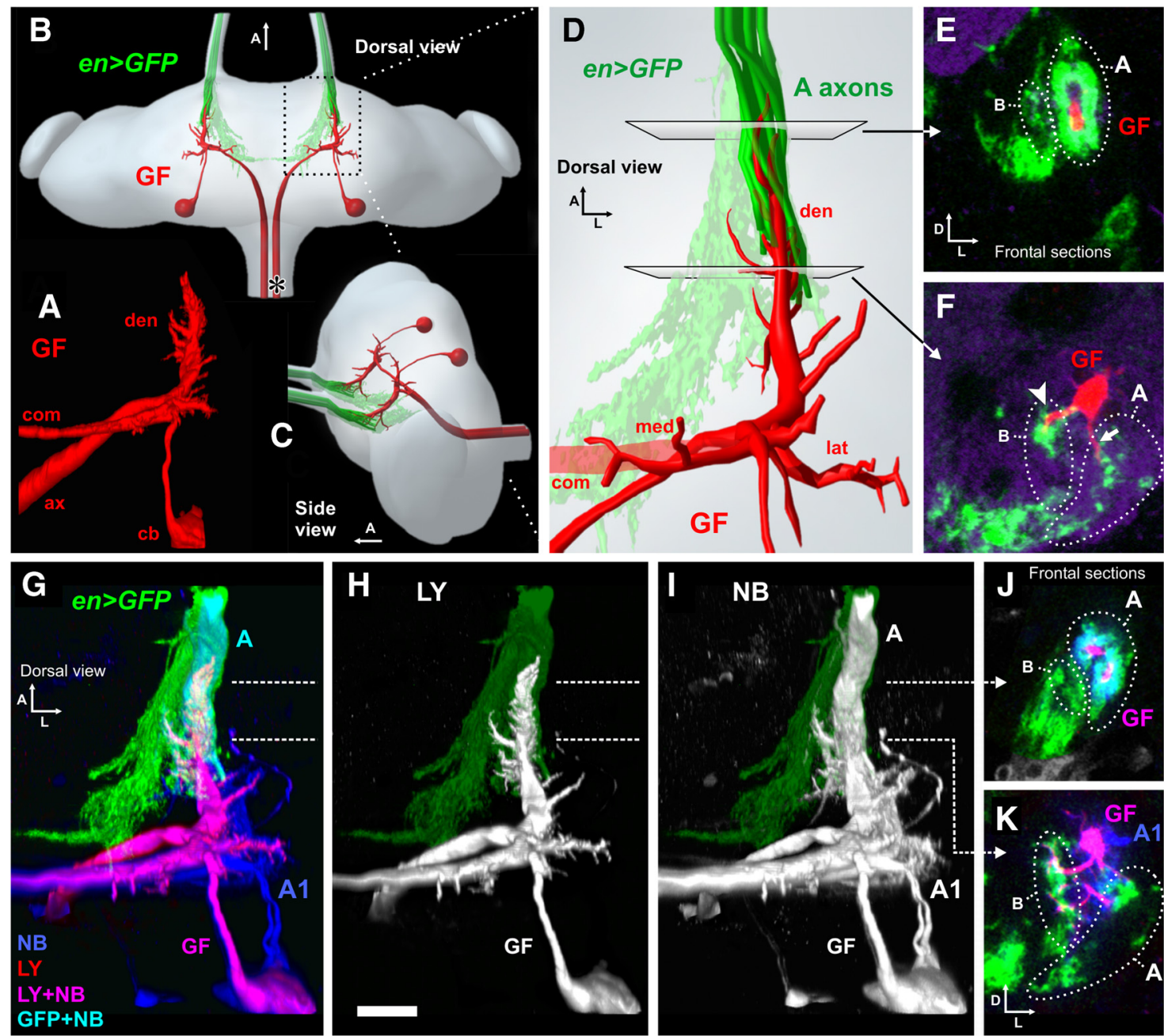

Figure 2. The GF dendrites are NB-coupled to some J0-A axons. In these animals, en-GAL4 was used to drive expression of CD8::GFP (genotype en-GAL4/UAS-CD8::GFP). $A$, The right GF was injected with $\mathrm{LY}$ (red) and viewed from the dorsal aspect. The cell body (cb), axon (ax), and dendrites (den) are indicated, along with LY-coupled processes of commissural neurons (com). $B$, $C$ Diagrammatic views of the brain, showing the pair of GF neurons (red) in relation to the antennal afferent projection (green). Dorsal $(\boldsymbol{B})$ and oblique ( $\boldsymbol{C}$ ) side views; arrows indicate anterior (A). The asterisk in $\boldsymbol{B}$ indicates the approximate site of GF axon impalement for dye injection. $\boldsymbol{D}$, Enlarged dorsal view of the GF dendrites, showing the anterior large dendrite (den) inserted within a cylinder of J0-A axons, and also the lateral (lat) and medial (med) dendrites. $\boldsymbol{E}$, Frontal confocal section (3 $\mu \mathrm{m}$ total) taken from the anterior end of the dendrite shown in $\boldsymbol{D}$, showing its insertion within a ring of several GFP-expressing J0-A afferents (A). A small number of JO-B afferents (B) are also stained. $\boldsymbol{F}$, Section taken more posteriorly along the GF dendrite, showing side branches approaching $A$ (arrow) and B (arrowhead) axons. G-I, Dorsal views of a complete confocal stack of a GF injected with LY (red) and NB (blue) in an animal expressing en-driven CD8::GFP. Superimposition of LY and NB within the GF makes it appear magenta. NB coupling is seen only within the cylinder of JO-A axons (cyan). GF is also NB-coupled to another interneuron (A1: blue). LY channel only ( $\boldsymbol{H}$ ) and NB channel only $(I)$ to better illustrate the NB coupling in the A axons.J, $\boldsymbol{K}$, Frontal confocal sections $(3 \mu \mathrm{m})$ through the GF dendrite, showing strong NB coupling in the A axons (cyan) but not in B axons. Scale bar (in $\boldsymbol{H}): \mathbf{G}-\mathbf{I}, 20 \mu \mathrm{m} ; \boldsymbol{E}, \boldsymbol{F}, \boldsymbol{J}, \boldsymbol{K}, 10 \mu \mathrm{m} . \mathrm{A}$, Anterior; D, dorsal; L, lateral.

burn, 2013). As described previously, using the nomenclature of Kamikouchi et al. (2006), axons of en-expressing JONs fall mainly into the JO-A and JO-E anatomical categories, with a few JO-Bs (Fig. 1F, G).

We took advantage of the visibility of the large GF axon within the cervical connective to inject it with the dye LY (Phelan et al., 1996; Boerner and Godenschwege, 2011). The GF forms a large dendrite that projects into the antennal mechanosensory and motor center (AMMC) of the neuropil. The dendrite extends in an anterior direction toward the point at which the antennal axons enter the brain (Fig. $2 A-C$ ). This dendrite is surrounded by a cylindrical cluster of $\sim 12-13$ En-expressing JO-A axons (Fig. $2 D, E)$, and, more posteriorly, its minor branches extend out in close proximity to some of the En-expressing JO-A and JO-B axons (Fig. $2 F$ ). None of the other lateral and medial dendrites of the GF come close to other AMMC areas. Therefore, we concur with previous observations that the GF arborizes only in the JO-A and JO-B zones of the AMMC neuropil (Kamikouchi et al., 2009; Lehnert et al., 2013).

Addition of the dye NB (287 Da molecular weight compared with $444 \mathrm{Da}$ for LY) to the injection microelectrode (Trimarchi and Murphey, 1997) shows that the GF is dye coupled selectively with NB (but not with LY) to the Enexpressing JO-A axons (Fig. 2G-J) and also to another interneuron projecting into the JO-A region (Fig. $2 G, K$ ), tentatively identified as AMMC-A1 (Kamikouchi et al., 2009; Tootoonian et al., 2012). It is clear that the JO axons are filled directly via the GF and not via the AMMC-A1 neuron, because the contralateral homolog of the latter also fills strongly yet on that side no JO axons are coupled to it (data not shown). No $\mathrm{NB}$ coupling is observed in the few En-expressing JO-B axons (Fig. $2 J, K)$. In a previous study, it appears that biocytin (372 $\mathrm{Da}$ molecular weight) is also apparently unable to pass from the GF to the JON axons (Lehnert et al., 2013), although this 

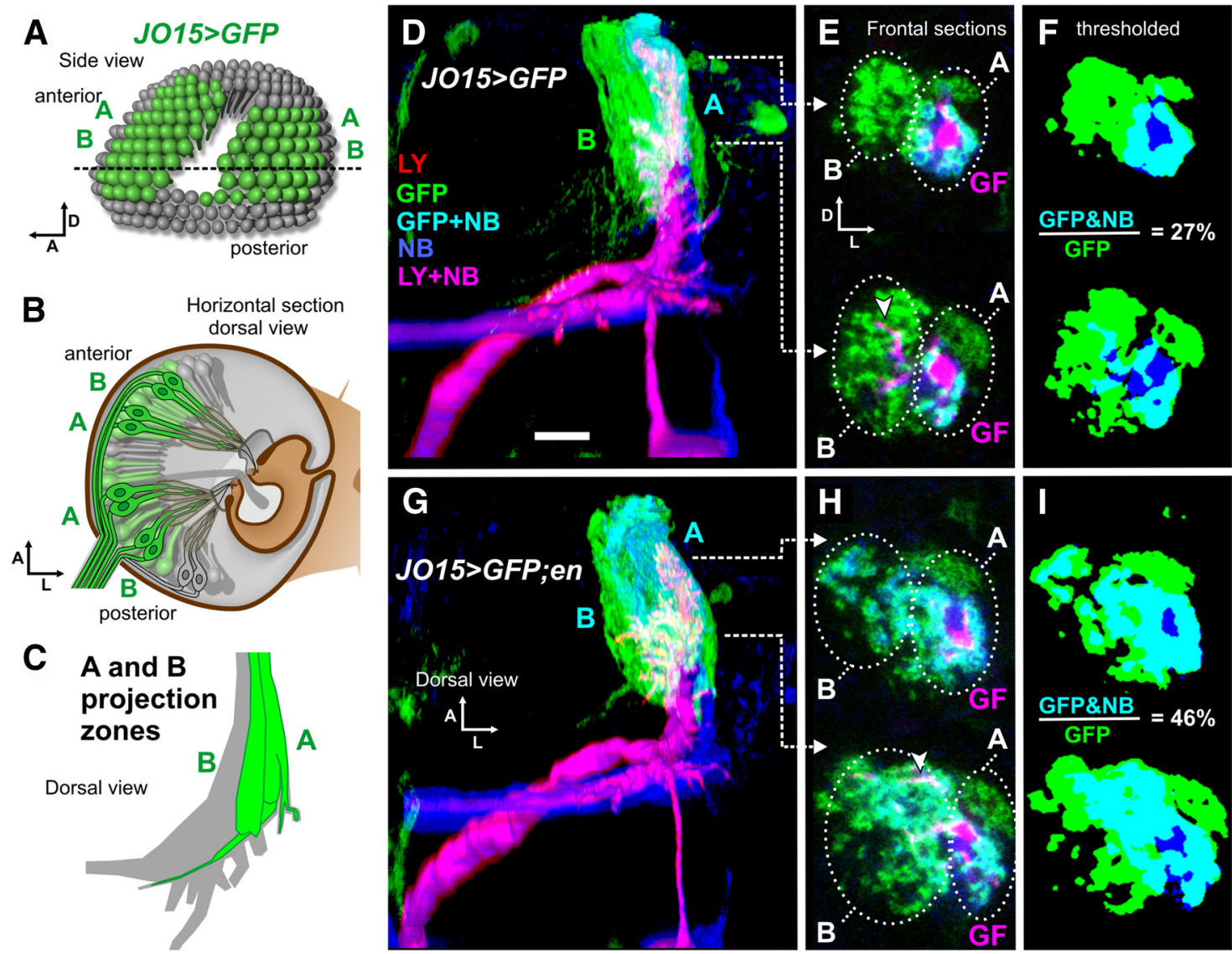

Figure 3. Ectopic en expression in JONs alters dye coupling to the GF. In these animals, JO-A and B JONs express CD8::GFP driven by the GAL4 line J015. Diagram showing the 3D arrangement of J015-expressing J0Ns of types A and B within the array of JON cell bodies $(\boldsymbol{A})$ and their position in a horizontal section of the pedicel $(\boldsymbol{B})$. $\boldsymbol{C}$, Diagram of $J 015$-expressing axonal projections in zones $A$ and B. D-F, Confocal images from control animals with genotype UAS-mCD8::GFP/+;J015-GAL4/+. D, Dorsal view of complete confocal stack from control animal, showing NB coupling in A axons only (cyan). $\boldsymbol{E}$, Single frontal confocal sections, taken from the regions indicated in $\boldsymbol{D}$. GFP-labeled $A$ and $B$ axons are indicated, along with the GF dendrite (magenta). Only the $A$ axons are NB-coupled (cyan), despite GF branches approaching B axons (arrowhead). $\boldsymbol{F}$, Thresholded representation of the same sections, showing the areas quantified. Dye coupling is expressed as the percentage ratio between the total area of intersection of blue and green channels (GFP \& NB) and the total area of the green channel (GFP). G-I, Confocal images from experimental animals with ectopic En expression (genotype UAS-mCD8::GFP/+;J015-GAL4/UAS-en). G, Dorsal view of complete confocal stack from experimental animals, showing NB coupling in A and B axons (cyan). $\boldsymbol{H}$, Single frontal confocal sections, taken from the regions indicated in G. GFP-labeled A and B axons are indicated, along with the GF dendrite (magenta). A and B axons are NB-coupled (cyan). GF branches approaching $B$ axons are indicated (arrowhead). I, Thresholded representation of the same sections. The area ratio of dye coupling has significantly increased (see Results). Scale bar (in D): $D, G, 20 \mu \mathrm{m} ; E, F, H, I, 10 \mu \mathrm{m}$.

may simply be attributable to a lower concentration in the recording electrode.

Ectopic expression of En in JO-B neurons causes them to NBcouple with GF

The En-expressing group of A-type JONs, which seem to be the only ones that are NB-coupled to the GF, make up only a small subset ( $\sim 10 \%)$ of the 145 sound-detecting JO-A and JO-B neurons (Kamikouchi et al., 2006). In view of the controlling effect of En on synaptic connectivity in the cockroach cercal system (Marie et al., 2000, 2002; Marie and Blagburn, 2003), we hypothesized that the expression of En could influence the formation of JON synapses with the GF. Therefore, we used the JO15-GAL4 line (Sharma et al., 2002), which is expressed in all of the JO-A and the majority of the JO-B neurons (Kamikouchi et al., 2006; Fig. 3A$C)$, to drive ectopic En expression in the latter group and then assessed whether NB coupling was altered (Fig. $3 D-I$ ).

In control animals, in which JO15-GAL4 was used to drive CD8::GFP expression alone, NB coupling was observed only in the JO-A axons, as described above; the GFP-labeled JO-B axons were not filled (Fig. 3D-F). However, when we used UAS-en to express En ectopically in these neurons, NB was seen to spread from the GF to their axons (Fig. 3G-I). The area of intersection, i.e., logical AND (indicated by \&), of NB and GFP signals (Fig. 3, cyan) was measured and averaged over a $30 \mu \mathrm{m}$ length of the JON axons, beginning with the anteriormost slice in which the GF dendrite appears (Fig. 3F,I). This area was expressed as a percentage of the total area of GFP signal. In experimental UAS-CD8::GFP/+;JO15-GAL4/UAS-en animals, there was a significant increase in the percentage area of NB-coupled axons compared with UAS-CD8::GFP/+;JO15-GAL4/+ controls (experimental, $46 \pm 2 \%, n=7$ vs control, $27 \pm 3 \%, n=5, p=$ $0.0037, t$ test). If it is assumed that the additional coupled JO-B axons have the same cross-sectional area as the JO-A axons and assuming that there is no great increase in axonal branching (for which we see no evidence), then ectopic En expression leads to at least a 70\% increase in the number of axons that synapse with GF. Not all JO-B axons couple with the GF; similarly, there is a group of dorsolateral JO15-expressing, normally En-negative, JO-A axons that also shows no coupling. Additionally, in control animals, there are a few JO-B and many JO-CE axons that express En but are also not coupled to the GF. There are probably additional factors besides En expression that determine whether JONs form gap junctions with the interneuron. 

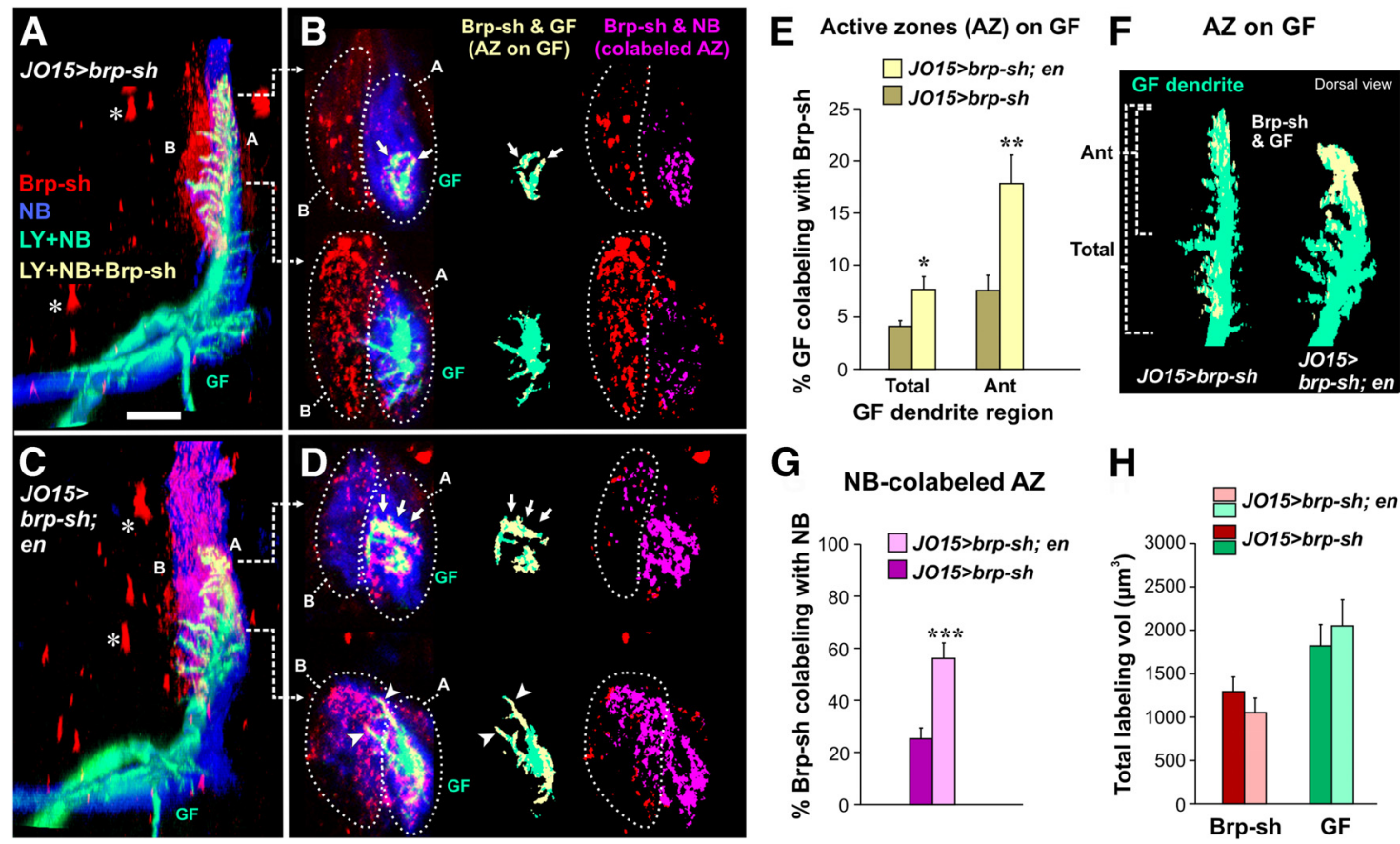

Figure 4. Ectopic expression of En increases the number of presynaptic sites on the GF. $A, B$, Control animal of genotype UAS-brp-sh-strawberry/+;J015-GAL4/+ in which the GF was injected with LY (green) and NB (blue). AZs in the JO-A and J0-B axons are labeled with Brp-sh (red). A, Dorsal view of complete stack, showing Brp-sh in J0-A and J0-B axons. Large agglomerations of Brp-sh are present in unidentified cell bodies (asterisks). $\boldsymbol{B}$, Projections of five frontal slices each from the preparation in $\boldsymbol{A}$, taken from the levels indicated. Left, JO-A (A) and J0-B (B) axons are encircled and the GF dendrite is indicated (cyan). NB (blue) is present in the GF and in the A axons. Puncta of Brp-sh (yellow: arrows) surround the GF dendrite. Middle, Quantification of AZs as the intersection of Brp-sh \& GF (yellow) compared with GF labeling (green). Right, Quantification of NB-colabeled AZs compared with Brp-sh labeling. C, D, Experimental animals of genotype UAS-brp-sh-strawberry/ $+; J 015-G A L 4 / U A S-$ en. D, Left and right, B axons show NB coupling (blue). Middle, There are more putative AZs on the GF dendrite (arrows). Bottom, There are more AZs on the medial branches of GF (arrowheads). Scale bar (in A): A, C, $20 \mu \mathrm{m} ; \boldsymbol{B}, \boldsymbol{D}, 10 \mu \mathrm{m}$. $\boldsymbol{E}, \mathbf{G}, \boldsymbol{H}$, Pooled data from 12 control flies (UAS-brp-sh-strawberry/+;J015-GAL4/+) and nine experimental flies (UAS-brp-shstrawberry/+;J015-GAL4/UAS-en). $\boldsymbol{E}$, The AZs on GF, expressed as the percentage of GF colabeling with Brp-sh by total volume. Values were calculated over the complete length of the GF dendrite (Total) and the anteriormost $30 \mu \mathrm{m}$ only (Ant). There are significant increases in the animals with ectopic En expression compared with controls. $\boldsymbol{F}$, Dorsal views of maximum intensity projections of thresholded GF dendrites (LY: green) with superimposed intersection of Brp-sh staining (yellow), showing the concentration of putative AZs in the anterior part of the dendrite and the increase with ectopic En expression. G, The percentage of Brp-sh colabeling with NB gives an estimate for the percentage of JO-A and JO-B axons that are NB dye-coupled. There is a significant increase in NB coupling in animals with ectopic En expression. $\boldsymbol{H}$, Total volume of labeling calculated over the length of the ROl for Brp-sh and GF staining. There is no significant difference in either measure between control animals and those with ectopic En expression.

\section{Ectopic expression of En in JO-B neurons causes them to} form more presynaptic active zones next to GF dendrites Clearly, the axons of En-expressing JO-B neurons are forming gap junctions with GF that did not exist before. Does this mean that their target recognition mechanisms have been altered, changing the specificity of their outputs? Or are the neurons already compatible synaptic partners, with chemical synaptic contacts present between them? In this case, En expression could simply alter the composition of these contacts, adding de novo gap junctions alongside the transmitter release machinery. Although electrical synapses can exist alone, mixed electrical/chemical seems to be a common morphological configuration, particularly in the GF system. Electron microscopy has shown that unidentified JON axon terminals form closely interspersed electrical and chemical synapses (Sivan-Loukianova and Eberl, 2005) and that other gap junctions onto the GF (from visual interneurons) are similarly accompanied by chemical contacts (Strausfeld and Bassemir, 1983). The GF itself forms mixed synapses with its targets (Blagburn et al., 1999). Some GF dendrites do normally project into the midst of JO-B axons without dye coupling with them (Fig. 3E, arrowhead), so they are appropriately located for this to happen.

We can distinguish between these two possibilities by taking advantage of this mixed synaptic composition and using a well characterized genetic marker for presynaptic active zones (AZs), a truncated version of the protein Brp coupled to a red fluores- cent dye Strawberry (Christiansen et al., 2011). Brp-sh-strawberry localizes reliably along with normal Brp at presynaptic AZs, although it does also accumulate in agglomerations within cell bodies (Christiansen et al., 2011). Thus, if synaptic recognition molecules have changed, we should see an increase in Brp-shlabeled AZs on the GF that parallels the increase in NB coupling to JO-B axons; conversely, if gap junctions are simply added to existing synapses, we should see no significant change in AZ distribution.

We first confirmed that Brp-sh expression is indeed a reliable marker for AZs in JO afferents. It colocalizes with CD8::GFP when driven by JO15-GAL4, and the Brp-sh puncta surrounding the GF dendrite stain with the nc82 antibody for the full-length native protein (data not shown). Some large agglomerations were also present in axons and small JO15-expressing cell bodies (Fig. $4 A, C$, asterisks), but these did not contain native Brp.

For these experiments, we used JO15-GAL4 to drive Brp-sh expression in JO-A and JO-B afferents and injected LY and NB into GF. Controls were of genotype UAS-brp-sh-strawberry/+; JO15-GAL4/+ (Fig. 4A,B), and experimental animals with ectopic En expression were of genotype UAS-brp-sh-strawberry/+; JO15-GAL4/UAS-en (Fig. 4C,D). As with the NB coupling experiments above, analysis of the confocal stacks began with one or two slices before the anteriormost slice in which the tip of the GF dendrite appeared and continued posteriorly through to the end of the Brp-sh fluorescence. Putative AZs abutting the GF 
were defined as the regions of colocalization of GF labeling and Brp-sh (Fig. $4 B, D$ ). The area of signal was summed over all of the slices to give an approximate volume and expressed as a percentage of the GF dendrite volume (\% GF colabeling with Brp-sh: Fig. $4 E$ ). In experimental animals with ectopic En expression, there was a doubling of this measure of putative AZs on the GF (Fig. 4E, Total; control, $4 \pm 1 \%, n=12$ flies vs experimental, $8 \pm 1 \%, n=$ 9 flies, $p=0.020, \mathrm{df}=11, t$ test for unequal variances). If we considered only the anteriormost 30 slices, in which most of the putative contacts are formed (Fig. $4 F$ ), this difference was even more pronounced (Fig. $4 E$, Ant; control, $8 \pm 1 \%, n=12$ flies vs experimental, $19 \pm 3 \%, n=9$ flies, $p=0.003, \mathrm{df}=12$, $t$ test for unequal variances).

The same preparations could be used to obtain an independent measure of NB coupling. Because the JO15-driven Brp-sh delineates the synaptic region of the JO-A and JO-B axons, we estimated NB coupling as that percentage of Brp-sh that colocalizes with NB (\% Brp-sh colabeling with NB; compare the right panels of Fig. $4 B, D$ ). In close agreement with our previous experiments (Fig. 3), there was a doubling in the percentage of axons with NB coupling (Fig. 4G; control, $26 \pm 5 \%, n=12$ flies vs experimental, $60 \pm 5 \%, n=9$ flies, $p=0.0014$, Mann-Whitney $U$ test).

Finally, to test whether En overexpression could simply be causing an overgrowth of the JO-A and JO-B axons and/or a general overproduction of synaptic components, we compared the total amount of Brp-sh labeling (Fig. $4 H$ ); there was no significant change (control, $1291.3 \pm 169.6, n=12$ flies vs experimental, $1079.3 \pm 163.6 \mu \mathrm{m}^{3}, n=9$ flies, $\mathrm{df}=19, p=0.392, t$ test). Similarly, there was no significant change in the total volume of the GF dendrite (Fig. $4 H$; control, $1818.9 \pm 246.4 \mu \mathrm{m}^{3}$, $n=12$ flies vs experimental, $2128.6 \pm 309.9 \mu \mathrm{m}^{3}, n=9$ flies, $\mathrm{df}=19, p=0.438, t$ test $)$

In conclusion, our result that the increase in putative AZs almost exactly parallels the increase in NB-coupled axons is most consistent with the first hypothesis; axons of En-expressing JO-B neurons have altered synaptic target recognition mechanisms that cause them to form new, mixed electrical and chemical synaptic contacts with GF.

\section{Ectopic expression of En in JO-B neurons results in a} redistribution of GF dendritic branches

In the course of the previous experiments, we observed that the main GF dendrite appeared to have more profuse medially directed branches in those animals in which En was driven with JO15-GAL4 (Fig. 5), although this did not correspond to a significant increase in dendrite volume overall. These dendritic branches were reconstructed from LY fills and their lengths were measured. There were in fact significant decreases in the number of laterally projecting GF dendrites within the 5-10 $\mu \mathrm{m}$ length category (control, $28.0 \pm 2.6, n=9$ flies vs experimental, $20.3 \pm$ 2.2, $n=11$ flies, $\mathrm{df}=18, p=0.036, t$ test) and the $25-30 \mu \mathrm{m}$ length category (control, $0.67 \pm 0.17, n=9$ flies vs experimental, $0.09 \pm 0.09, n=11$ flies, $\mathrm{df}=18, p=0.009$, Mann-Whitney $U$ test), and significant increases in the numbers of medially projecting dendrites, specifically those in the very short $(0-5 \mu \mathrm{m}$ : control, $39.7 \pm 4.2, n=9$ flies vs experimental, $53.5 \pm 4.1, n=11$ flies, $\mathrm{df}=18, p=0.032$, $t$ test $)$, medium $(15-20 \mu \mathrm{m}$ : control,
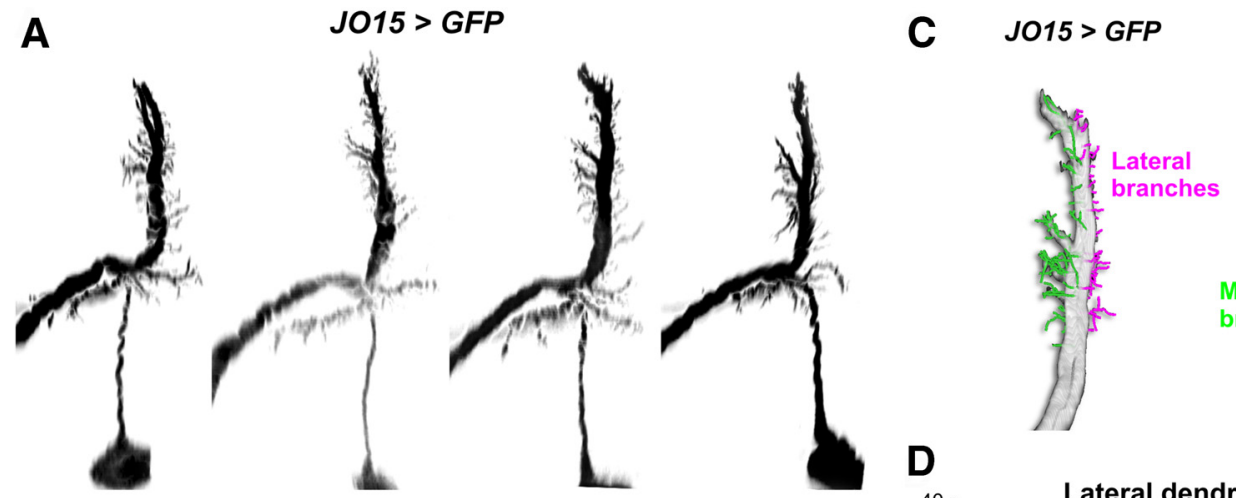

J015 > GFP; en

B

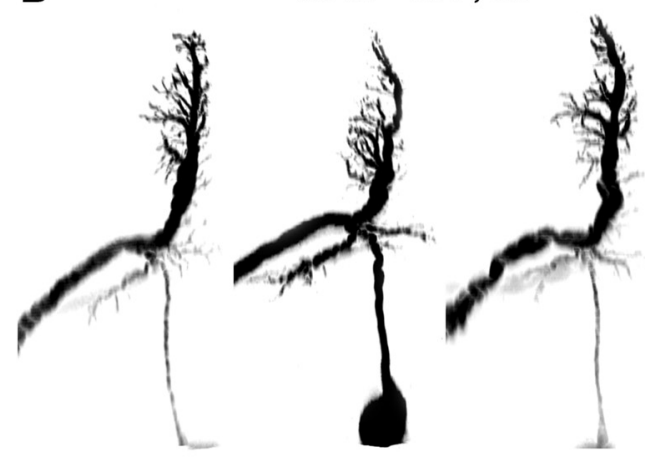

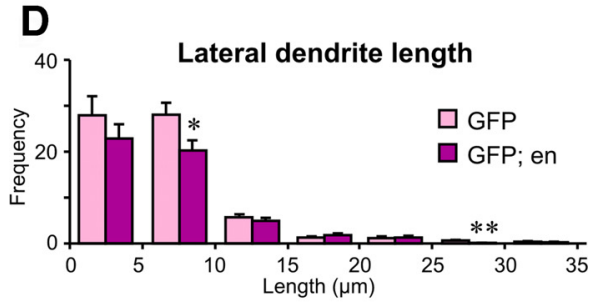

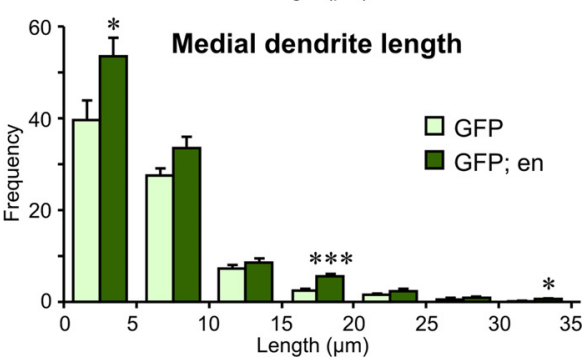

Figure 5. Ectopic synapse formation leads to the GF having longer medial branches. $\boldsymbol{A}$, Morphology of the GF in control animals (dorsal views of maximum intensity projections, shown in negative). $\boldsymbol{B}$, GF dendrites in animals with ectopic En expression appear to have more medial branches. $\boldsymbol{C}$, Length tracing of lateral (magenta) and medial (green) GF dendrites. $\boldsymbol{D}$, With ectopic En expression in JONs, there are significant decreases in the number of lateral dendrites in short and long classes and significant increases in the number of short, medium, and long medial dendrites. 
$2.44 \pm 0.47, n=9$ flies vs experimental, $5.55 \pm 0.55, n=11$ flies, $\mathrm{df}=18, p=0.0006, t$ test $)$, and long $(30-35 \mu \mathrm{m}$ : control, $0.11 \pm$ $0.11, n=9$ flies vs experimental, $0.64 \pm 0.20, n=11$ flies, $p=$ 0.046 , Mann-Whitney $U$ test) categories (Fig. $5 D$ ). It is reasonable to conclude that the formation of additional synaptic connections with the more medially located JO-B axons results in the enhancement of the growth of medial GF dendritic branches, at the expense of the lateral ones.

\section{Electrophysiological measurement of JON inputs to the GF}

The GF escape circuit (Fig. 6A) is the first neuronal circuit in Drosophila that was found to be amenable for electrophysiology (Levine and Tracey, 1973; Tanouye and Wyman, 1980; Thomas and Wyman, 1984; Allen et al., 1999; Allen and Godenschwege, 2010). In addition to the mechanosensory inputs from the JO that we are investigating here, the GF is known to receive synaptic inputs from the visual system onto its lateral dendrites (Bassemir and Strausfeld, 1983; Mu et al., 2012). Looming or darkening visual stimuli (Card and Dickinson, 2008; von Reyn et al., 2014), coincident with air movements detected by the JO, are optimal for evoking the fly's escape response (Hammond and O'Shea, 2007). The GF axon descends to the thoracic ganglion (Fig. 6A), in which it forms mixed electrical and chemical synapses with the peripherally synapsing interneuron (PSI) and the TTM motoneuron (TTMn; Blagburn et al., 1999).

In wild-type animals, an action potential in the GF invariably triggers a contraction in first the TTM then, $\sim 0.4 \mathrm{~ms}$ later, one in the DLM (Tanouye and Wyman, 1980). These muscle action potentials serve as a reliable readout for the occurrence of an action potential in the GF itself (Allen and Godenschwege, 2010). In many studies, the GF is stimulated directly with a strong voltage stimulus across the eyes (Fig. 6A), but here we use the "longlatency" (3-5 ms) response of the GF, elicited by a weak stimulus, that is attributable to stimulation of the polysynaptic visual pathways (Engel and Wu, 1996; an example can be seen in Fig. 6B4). Although it receives direct synaptic input from the JONs, auditory stimuli alone are apparently not sufficient to depolarize the GF above threshold for spike initiation (Fig. 6B1-B3). Therefore,
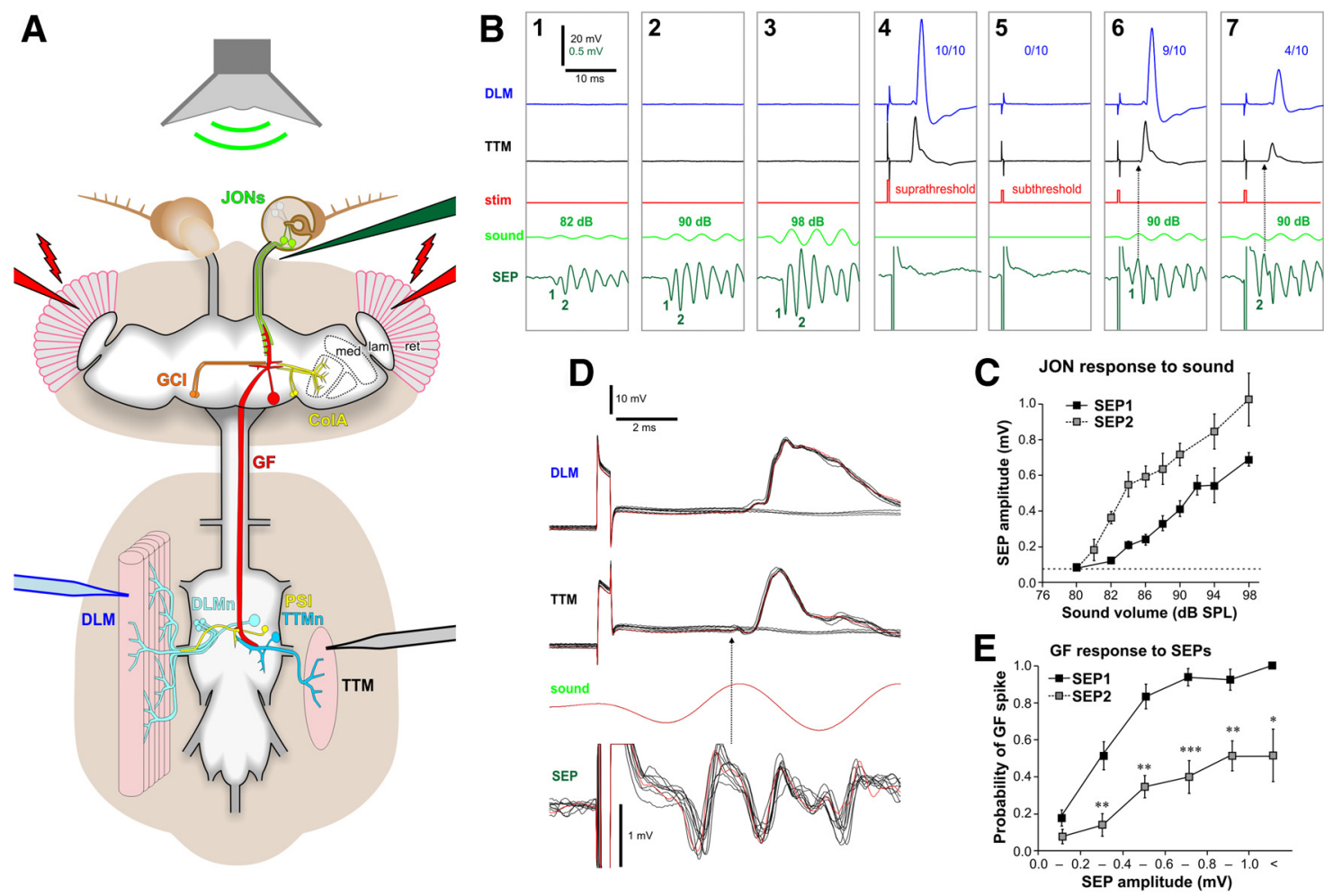

Figure 6. The strength of the synapse between JONs and the GF can be monitored through indirect recording of the GF system. $A$, Diagram of the GF escape circuit and position of the recording electrodes. In the brain, the GF receives synaptic inputs onto its dendritic branches from the mechanosensory JONs and from polysynaptic visual pathways (ret, retina; lam, lamina; med, medulla; ColA, lobular columnar type A interneurons). It also forms synaptic connections with the GCI. The GF axon descends to the thoracic ganglion in which it forms electrical and chemical synapses with the TTMn of the TTM and the PSI, which innervates the dorsal longitudinal motoneurons (DLMn) of the DLM. A low-voltage stimulus across the eyes (red electrodes) was used to stimulate the visual pathway. A $200 \mathrm{~Hz}$ sine wave sound stimulus (green) delivered with a speaker was used to stimulate the JONs. SEPs were recorded from the antennal nerve with an electrode (dark green) inserted at the base of the antenna. The output of the GF pathway was recorded from the TTM (black electrode) and the DLM (blue electrode). B1-B7, Averages of 10 recording traces. B1-B3, Sounds of increasing volume give progressively larger SEPs in the antennal nerve, which fail to activate the GF. Note the gradual sound onset. $B$ 4. Strong voltage stimulus (suprathreshold) depolarizes the GF above threshold, resulting in action potentials in both DLM and TTM in all 10 trials ( 10 of 10$)$. B5, For subsequent experiments, the voltage stimulus was adjusted so that the GF was just under its threshold for action potentials (subthreshold). Note the large stimulus artifacts in the antennal nerve traces in B4-B7. B6,A $90 \mathrm{~dB}$ sound stimulus summates with the subthreshold voltage stimulus to activate the GF. The GF fires an action potential in 9 of 10 traces, making the averaged amplitudes of the TTM and DLM spikes lower than those in $B 4$. The delay of the voltage stimulus was adjusted to investigate the GF response to SEP1. B7, The stimulus delay was increased to investigate SEP2. The GF response occurs in only 4 of 10 traces, despite the larger SEP 2 amplitude, making the averaged amplitude even smaller. C, Mean SEP amplitude versus sound volume for controls (JO15-GAL4/+, $n=10$ flies), showing that both SEPs increase linearly with volume and that for a given volume SEP1 is smaller than SEP2. Dotted line indicates the level of background activity in the antennal nerve. $\boldsymbol{D}$, Example traces from 10 trials with synchronized sound and subthreshold voltage stimuli. The most recent trial is shown in red. In 7 of 10 traces, the GF fires an action potential in response to SEP1 (responses from both TTM and DLM). In 3 of 10 trials, the GF failed to fire an action potential (no muscle response). Dashed arrow shows the start of the TTM response synchronized with the end of SEP1. $E$, The GF response to SEPs of increasing amplitudes (a measure of the strength of the synapse between JONs and GF). An increase in amplitude of SEP1 gives an increase in GF spike probability. SEP2 shows significantly less excitatory effect on GF, at all amplitudes (J015-GAL4/+ , $n=$ 10 flies, Mann-Whitney $U$ test). 
we used a subthreshold stimulus across the eyes to stimulate the visual inputs and depolarize the GF close to its threshold for action potentials, thus enabling spike initiation with a coincident auditory stimulus (Fig. 6B6,B7). This provided a measure of synaptic input from the sound-sensitive JONs. The GF firing was monitored via the recording of TTM and DLM. Although this is an admittedly indirect measure of synaptic inputs that could otherwise be measured directly by intracellular recording ( $\mathrm{Mu}$ et al., 2012; Tootoonian et al., 2012; Lehnert et al., 2013), it has several advantages. It is technically easy, allowing for very long duration experiments, and, importantly, it permits the simultaneous extracellular recording of presynaptic SEPs with a tungsten electrode inserted into the base of the antenna.

For the sound trials, we used pure tones of $200 \mathrm{~Hz}$ because these elicit strong, well defined, responses in En-expressing JONs (Pézier and Blagburn, 2013). Above a threshold of $\sim 80 \mathrm{~dB}$ sound pressure level, the amplitude of the SEPs increased almost linearly with sound volume (Fig. $6 \mathrm{C}$ ). It should be noted that the amplitude of SEP1 is always $0.66 \times$ smaller than that of SEP2 at any given volume; this is simply attributable to the fact that the sound stimulus was designed to have a gradual onset to prevent a JO response to transient air acceleration (Fig. 6B1-B3,D).

By altering the delay of the visual stimulus, we were able to synchronize it with either the first or the second SEP (Fig. 6, compare $B 6, B 7)$. This enabled us to investigate the response of the GF to synaptic input from each of these presynaptic compound potentials in turn. An example experiment is shown in Figure $6 D$, in which the GF activates TTM and DLM in 7 of 10 trials in response to the subthreshold visual stimulus being paired with SEP1, i.e., has a firing probability of 0.7 . For SEP1, the mean probability of the GF firing increased approximately linearly with presynaptic spike amplitude, plateauing at the maximum of 1.0 for amplitudes $>0.6 \mathrm{mV}$ (Fig. $6 E$ ).

Strangely, the GF was much less sensitive to SEP2s of any amplitude (Fig. $6 E$ and example in B7; Mann-Whitney $U$ test: bin $0.0-0.2 \mathrm{mV}, p=0.1240, n=10 \mathrm{SEP} 1$ and $n=8 \mathrm{SEP} 2$; bin $0.2-0.4 \mathrm{mV}, p=0.0058, n=10 \mathrm{SEP} 1$ and $n=8 \mathrm{SEP} 2$; bin $0.4-0.6 \mathrm{mV}, p=0.0013, n=10 \mathrm{SEP} 1$ and $n=8$ SEP2; bin $0.6-0.8 \mathrm{mV}, p=0.0007, n=10 \mathrm{SEP} 1$ and $n=8$ SEP2; bin $0.8-1.0 \mathrm{mV}, p=0.0032, n=9 \mathrm{SEP} 1$ and $n=7 \mathrm{SEP} 2$; bin $>1 \mathrm{mV}$, $p=0.0229, n=4$ SEP1 and $n=6$ SEP2). At present, we have no definitive explanation for this observation. It cannot be attributable to spike adaptation in the GF, because no action potentials are elicited in it by sound alone. One explanation could be rapid spike rate adaptation in the JO-A afferents, as suggested previously (Lehnert et al., 2013), in which case a substantial part of SEP2 must represent the action potentials of a non-GF-coupled population of afferents, which fire too late to contribute to SEP1. Alternatively, there could be an inhibitory interaction between SEP1 and SEP2 (or the responses of the GF to them). A role for chemical synaptic release in this is ruled out by tetanus toxin expression experiments (see below). Other possible mechanisms could involve rectification of the gap junctions, or fluctuations in the threshold of the GF. Clearly, more detailed experiments, coupled with intracellular GF recording, are necessary to elucidate this phenomenon. However, the important point here is that, whatever the precise mechanism involved, this effect on the response of the GF to SEP2 is affected by perturbation of En expression (see below).

\section{Ectopic En expression strengthens the JON-GF connection} Because the ectopic expression of En in the B group of JONs alters the pattern of dye coupling and distribution of synaptic connec- tions, we investigated the effects of this treatment on the synaptic input to the GF. In cockroach sensory neurons, the paralog of En, Inv, has subtly different effects on connectivity (Marie and Blagburn, 2003). We were interested to see whether these differences are conserved in Drosophila so we also tested the effects of its overexpression in JONs, using the EP line $P\{E P\}-i n v^{G E 10665}$, which has Gal4 binding sites (UAS) inserted in the inv gene (Rørth, 1996). Using the electrophysiological assays described above, experimental animals of genotype JO15-GAL4/UAS-en or JO15-GAL4/P\{EP\}-inv ${ }^{G E 10665}$ were compared with controls (JO15-GAL4/+). Importantly, there were no significant differences in SEP amplitude across the three treatments (Fig. $7 A, B$ ), so JON excitability is not affected (SEP1, $p=0.894$ at $82 \mathrm{~dB}$, $p=0.970$ at $90 \mathrm{~dB}, p=0.727$ at $98 \mathrm{~dB}$; SEP2, $p=0.175$ at $82 \mathrm{~dB}$, $p=0.493$ at $90 \mathrm{~dB}, p=0.122$ at $98 \mathrm{~dB}, \mathrm{df}=2$, one-way ANOVA; JO15-GAL4/+, $n=15 ;$ JO15-GAL4/UAS-en, $n=13$; $P\{E P\}$ inv ${ }^{G E 10665} /+; J O 15-G A L 4 /+, n=7$ flies). However, the response of GF to these SEPs was increased by ectopic En expression. For SEP1, there was a significant $\sim 40 \%$ increase in the likelihood of the GF firing in response to small SEP amplitudes of 0.2-0.4 mV (Fig. 7C; JO15-GAL4/+, $n=10$; JO15-GAL4/UASen, $n=10$ flies, $p=0.045$, Mann-Whitney $U$ test).

An even stronger effect for En expression was seen for SEP2, in which the GF responses increased to the point where they were not significantly different from those to SEP1 (Fig. 7D; from smaller to higher SEP amplitude bins, $p=0.0345, p=0.0007$, $p=0.0010, p=0.0044, p=0.0856, p=0.3865$, Mann-Whitney $U$ test; JO15-GAL4/+, $n=10 ; J O 15-G A L 4 / U A S-e n, n=10$ flies). Incidentally, none of these phenomena are dependent on chemical synaptic release, because expression of tetanus toxin light chain (TeTxLC; Sweeney et al., 1995) in JO-A and JO-B axons of both control and experimental animals did not alter any of our results (data not shown). Whatever the mechanism behind the lower control SEP2 response, the addition of inputs from JO-B neurons presumably accounts for this increase in response strength.

Overall, the results for SEP1 and SEP2 are wholly consistent with our previous observations that sound-sensitive JO-B axons form de novo synaptic contacts (mixed electrical and chemical) with the GF, thus increasing the strength of the synaptic connection. It is also possible that expression of larger amounts of En protein in JO-A neurons (Fig. $7 F$ ) results in them making a stronger synaptic connection with GF.

In contrast to En, ectopic Inv expression in JONs had no significant effects (Fig. 7C,D; Mann-Whitney $U$ test, JO15-

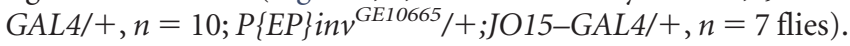
Assuming that the EP line is functional, this could perhaps mean that the two paralogs have different regulatory targets. This line is certainly able to express Inv protein in the antenna when driven by JO15-GAL4. Immunocytochemistry of control pupal antennae $[\sim 30 \mathrm{~h}$ after pupal formation (APF) $]$ with antibodies against En and En+Inv (mAb 4D9) shows that, in controls, the paralogs are colocalized in the nuclei of JO15-expressing JONs in the posterior part of the antennal pedicel (Fig. $7 E$ ) and that only one neuron in the anterior portion expresses the proteins (Fig. 7E, inset). Using JO15-GAL4 to drive UAS-en is clearly very effective at inducing large amounts of En protein (staining with both antibodies appears magenta in Fig. $7 F$ ). The inv EP line also works, albeit less effectively (Fig. 7G). More JONs are stained blue (Inv or En) in the anterior part of the antenna. However, there are several JONs that are not stained, despite having strong enough Gal4 expression to produce moderate GFP fluorescence and to drive UAS-en strongly (Fig. 7, compare insets in $F$ and $G$ ). The 


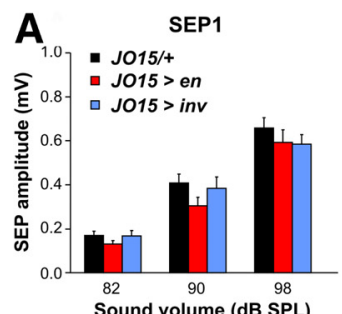

Sound volume (dB SPL)

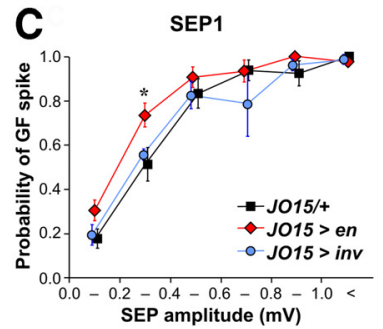

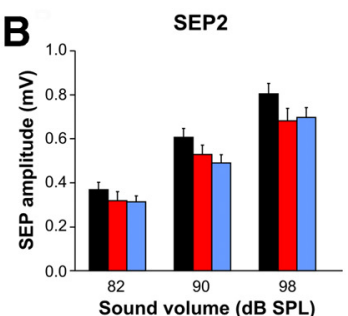

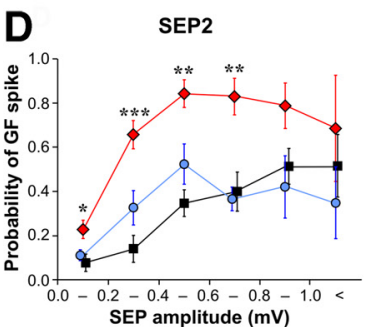

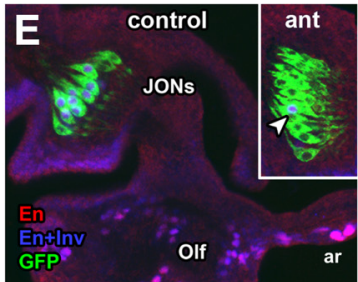
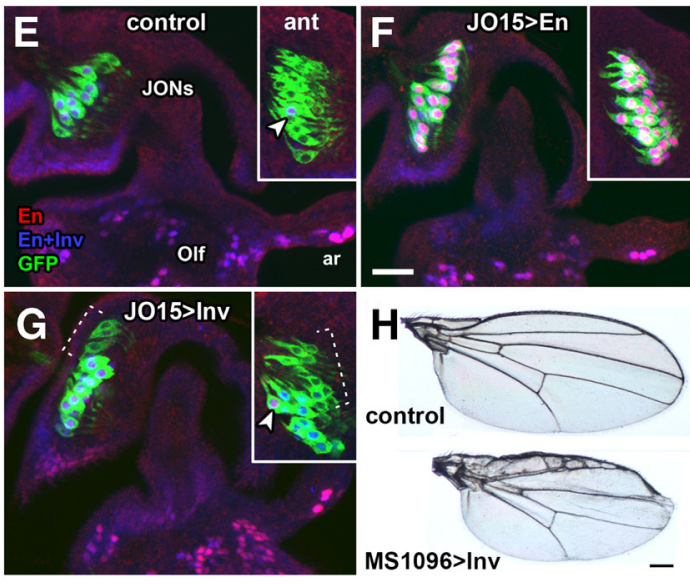

Figure 7. Ectopic En expression increases JON excitation of GF. $A, B, S E P 1$ and SEP2 amplitudes in control animals (genotype J015-GAL4/+), in animals with ectopic En expression (J015-GAL4/ UAS- en), and in animals with ectopic Inv expression (P\{EP\}inv $\left.v^{G E 10665} /+; J 015-G A L 4 /+\right)$. There are no significant changes in the relationship between SEP amplitude and sound volume between the different treatments (one-way ANOVA). C, D, Probability of eliciting a GF action potential for SEP1 and SEP2. For SEP1, ectopic En expression significantly increases the GF response to medium amplitude SEPs. For SEP2, ectopic En expression significantly increases the GF response to SEPs of all amplitudes (Mann-Whitney $U$ test). E-G, Immunolocalization of En and Inv proteins in $30 \mathrm{~h}$ APF pupal antennae. All animals express CD8::GFP driven by J015-GAL4. Anti-En staining is shown in red and 4D9 staining (En + Inv) in blue (Costaining with both antibodies ranges from purple to magenta). JONs, olfactory neurons (OIf), and aristal sensory neurons (ar) are indicated. The main figures show slices from the posterior region of the antenna, and the insets show anterior slices. $\boldsymbol{E}$, Control animals expressing CD8::GFP only (UAS-mCD8::GFP/+;J015-GAL4/+). There is moderate anti-En and 4D9 staining in a group of posterior J0Ns and in only a single neuron of the anterior group of JONs (arrowhead). $F$, In animals expressing ectopic En (UAS- $m$ CD8::GFP/+;J015-GAL4/UAS- en), there is strong labeling with both antibodies in both posterior and anterior J0N groups. G, In animals expressing ectopic Inv (UAS-mCD8::GFP/P\{EP\}inv $\left.{ }^{G E 10665} ; J 015-G A L 4 /+\right)$, there is some additional labeling with the 4D9 antibody (En + Inv) in both posterior and anterior J0N groups, but some neurons show no expression (dashed brackets). The single En- and Inv-positive neuron in the anterior group is indicated (arrowhead). $\boldsymbol{H}$, Driving the inv EP line in the wings with $B X^{M S 1096}$ GAL4 results in perturbation of the anterior compartment. Scale bars (in $\boldsymbol{F}$ and $\boldsymbol{H}$ ): $\boldsymbol{E}-\boldsymbol{G}, 20 \mu \mathrm{m} ; \boldsymbol{H}, 200 \mu \mathrm{m}$.

Inv protein produced by the EP line does seem to be functional, because expressing it in wing discs with $B x^{M S 1096}-G A L 4$ consistently results in defects in the anterior compartment of the wing (Fig. $7 H$ ). In fact, the site of the insertion of the EP line may result in one of the inv transcripts having a truncated $5^{\prime}$-UTR, which could perhaps account for the reduced expression pattern that we observe (Pfeiffer et al., 2012). Thus, the lack of effect of ectopic Inv expression on the JON-GF connection could be attributable simply to its expression being driven at lower levels in the JONs compared with En rather than to any specific differences in target genes between the two transcription factors.

\section{En and Inv knockdown decreases SEP amplitude and reduces} the JON-GF connection

What is the effect of removing En from the JONs that normally express it? Because we needed to remove expression of both En and Inv, which probably have overlapping functions, from most or all of the JONs to perform our assays and because loss of en and inv in large clones of the developing antenna leads to profound disruptions in patterning (Morata et al., 1983), we were unable to use mosaic analysis techniques to investigate this. Instead, we used RNA interference (RNAi), along with Dicer-2 (Dcr-2) expression, to knock down expression of both genes.

Unfortunately, JO15-GAL4 could not be used to drive RNAi because it had unexpected inhibitory effects on the SEPs when combined with UAS-Dcr-2. Instead, we used the driver pebGAL4, which expresses strongly in all postmitotic antennal sensory neurons (Sweeney et al., 2007). This is in fact an even stronger driver in the JONs than JO15-GAL4 and has the additional advantage of being present earlier in development. GFP expression driven by peb-GAL4 is already present in JONs just before antennal eversion at $\sim 10 \mathrm{~h} \mathrm{APF}$ (our unpublished observations), at which time some JON axons have already entered the brain (Lienhard and Stocker, 1991). Whether they have encountered the dendrite of the GF by this time is not clear (Allen et al., 1998). With the inclusion of Dcr-2 and raising the animals at $30^{\circ} \mathrm{C}$, substantial knockdown of both En and Inv could be achieved, as judged from qualitative immunocytochemistry of 30-40 h APF antennae (Fig. 8A,B). Unfortunately, doubleknockdown animals kept at $30^{\circ}$ or even $25^{\circ} \mathrm{C}$ rarely eclosed successfully, showing evidence of defects in head formation. To obtain sufficient experimental animals for electrophysiology, we had to raise the pupae at $20^{\circ} \mathrm{C}$, resulting in incomplete knockdown of En and Inv in the JONs (data not shown).

Surprisingly, the most obvious effect of this partial En and Inv knockdown was to decrease the amplitude of the SEPs to $\sim 75 \%$ of control values (Fig. $8 C, D$; SEP $1, p=0.185$ at $82 \mathrm{~dB}, p=0.029$ at $90 \mathrm{~dB}, p=0.006$ at $98 \mathrm{~dB}$; SEP2, $p=0.0037$ at $82 \mathrm{~dB}, p=$ 0.0043 at $90 \mathrm{~dB}, p=0.0009$ at $98 \mathrm{~dB}, \mathrm{df}=25$, unpaired $t$ test with equal variance or Mann-Whitney $U$ test; peb-GAL4/+;;UASDcr-2/+, $n=13 ;$ peb-GAL4/+;UAS-inv-RNAi/+;UAS-en$R N A i / U A S-D c r-2, n=14$ flies). This result confirms that the RNAi is at least partially functional in the JONs and, more importantly, suggests that En and Inv are involved in regulating the transcription of molecules that transduce the response to sound and/or promote neuronal excitability.

Because of the decrease in SEP amplitudes in knockdown animals, higher sound volumes were required to elicit SEPs of the appropriate amplitude to compare with controls. Even so, it was not possible to evoke the largest SEPs, so in the plots of GF firing probability versus amplitude (Fig. $8 E, F$ ), the $>0.7 \mathrm{mV}$ point for SEP1 and $>1.0 \mathrm{mV}$ point for SEP2 could not be obtained. Nevertheless, these plots show that, within the limits of our assay, there was no effect of double en and inv knockdown on the GF response to SEP1 (Fig. 8E). However, the response to SEP2 was significantly reduced for SEP amplitudes in the $0.6-0.8 \mathrm{mV}$ 

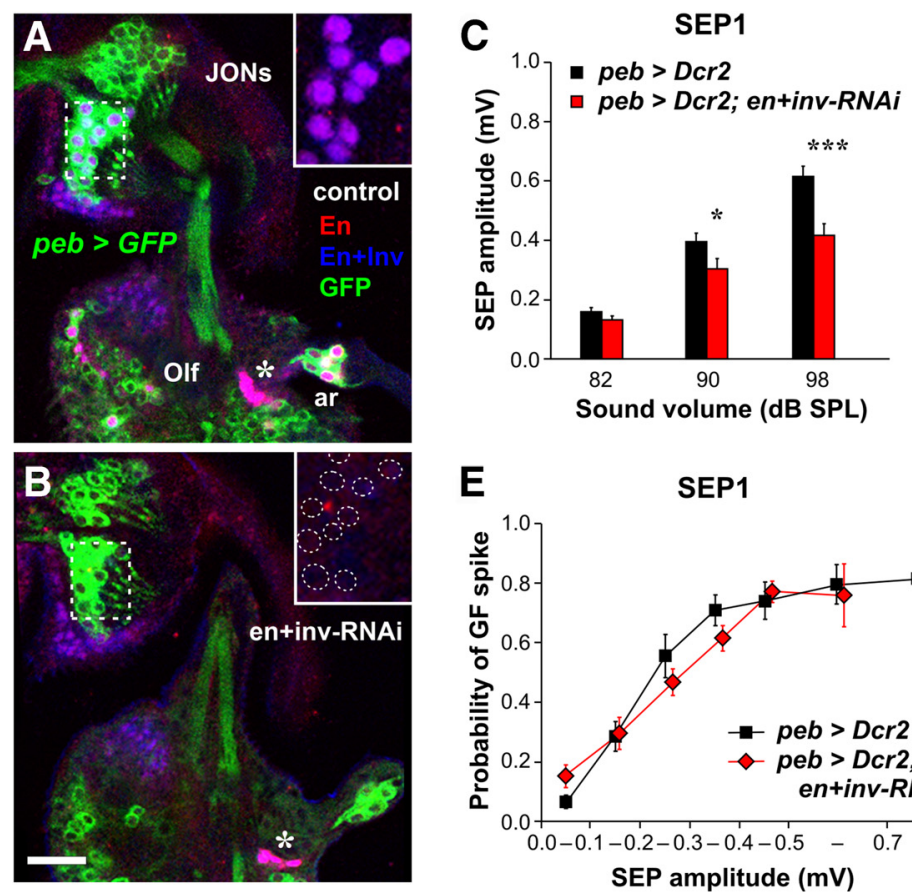

$E$
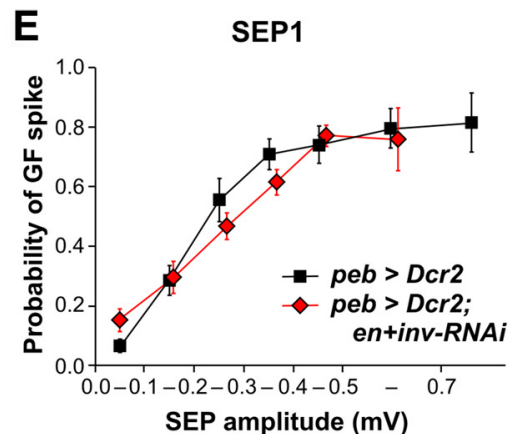

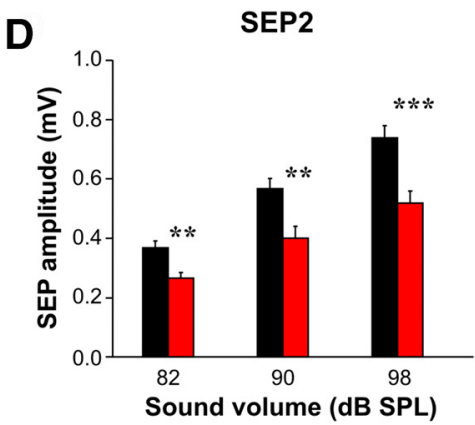

$\mathbf{F}$

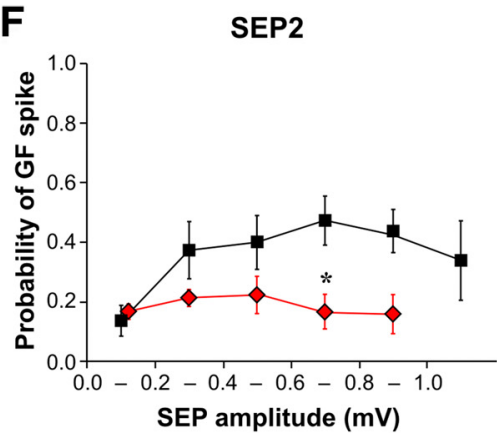

Figure 8. En knockdown with RNAi. A, D, Immunolocalization of En and Inv proteins in $30 \mathrm{~h}$ APF pupal antennae of animals with CD8::GFP driven by peb-GAL4. Anti-En staining is shown in red and 4D9 staining (En + Inv) in blue. J0Ns, olfactory neurons (Olf), and aristal sensory neurons (ar) are indicated. The main figures show slices from the posterior region of the antenna, and the insets show enlarged views of antibody staining only. $\boldsymbol{A}$, In control animals of genotype peb-GAL4,UAS-mCD8::GFP/+ ;UAS-DCr-2/+, there is anti-En and 4D9 staining in some of the JONs and strong staining in olfactory neurons. One cluster of En- and Inv-positive cells does not express peb-GAL4 (asterisk). $\boldsymbol{B}$, In double-knockdown RNAi animals of genotype peb-GAL4,UAS-mCD8::GFP/+ ;UAS-DCr-2/UAS-inv-RNAi;UAS- en-RNAi/+, there is no detectable anti-En or 4D9 immunoreactivity in GFP-expressing JONs, olfactory neurons, or aristal neurons. C, D, SEP1 and SEP2 amplitudes in control animals ( peb-GAL4/+,;UAS-DCr-2/+, $n=13$ ) and animals with knockdown of En and Inv in the antenna ( $p$ eb-GAL4/+,UAS-inv$R N A i /+; U A S-$ en-RNAi/UAS-Dcr-2, $n=14$ ), showing a decrease in SEP amplitudes in knockdown animals ( $t$ test). $\boldsymbol{E}, \boldsymbol{F}$, Probability of eliciting a GF action potential for SEP1 and SEP2. Double en and inv knockdown reduces the strength of the JON-to-GF synaptic connection but only for SEP2 at $0.6-0.8 \mathrm{mV}$ (Mann-Whitney $U$ test; control flies, $n=11$; RNAi knockdown flies, $n=7$ ). Scale bar (in $\boldsymbol{B}$ ): $\boldsymbol{A}, \boldsymbol{B}, 20 \mu \mathrm{m}$; insets, $10 \mu \mathrm{m}$.

range $(p=0.018$, Mann-Whitney U test; peb-GAL4/+;UASDcr-2/+, $n=11 ;$ peb-GAL4/+;UAS-inv-RNAi/+;UAS-en$R N A i / U A S-D c r-2, n=7$ flies that reached that amplitude range of 12 total knockdown flies tested).

In view of the inhibitory effects of double en and inv knockdown on the synapse, we performed NB injections in two animals to see whether dye coupling was affected; qualitative observations suggested that it was not, so this was not pursued further. For technical reasons, we were unable to make flies expressing UASbrp-sh-strawberry in addition to the RNAi components, so we were also unable to investigate whether putative synaptic contacts are reduced in numbers.

Because peb-GAL4 only drives expression in postmitotic neurons, it is possible that knockdown of en and inv would be more effective if done in the precursors of the JONs. Therefore, we also tried the ato-GAL4 driver, which is expressed in all JO precursors but is absent from early neurons, and then appears again in a subset of JONs late in pupal development (Roy et al., 2013). In animals raised at $25^{\circ} \mathrm{C}$, we found no significant effects on either SEP amplitude or the JON-GF connection (data not shown). We were unable to test animals raised at $30^{\circ} \mathrm{C}$ because the funiculus was distorted and unable to move freely, preventing the response to sound (although confirming that a greater degree of en/inv knockdown takes place).

\section{Discussion}

We show here that there is dye coupling from the GF into a subset of sound-detecting JONs that express the transcription factor En and its paralog Inv. We only see significant coupling with NB and not with LY, suggesting that these gap junctions have a smaller pore diameter, and therefore a different composition, than those between the GF and its postsynaptic targets TTMn and PSI, which readily dye couple with LY (Phelan et al., 1996). Moreover, it is only a subset of JO-A axons that dye couple with GF; other En-expressing JO-A, JO-B, and JO-E axons do not. In addition to being present at the JON synapses, ultrastructural gap junctions are also observed between the JON axons themselves (SivanLoukianova and Eberl, 2005). If so, our result implies that either those inter-axonal junctions are present between all JON axons but are incapable of passing NB or that, if they can pass NB, they must be restricted to the JO-A axons.

We find that ectopic expression of En in the JO-B neurons leads them to become NB-coupled with the GF. This could be attributable to En promoting the formation of new gap junctions at existing synapses; it is known that En positively regulates the netrin receptor Frazzled (Joly et al., 2007), which in turn regulates the expression of innexins in the GF (Orr et al., 2014). However, we also show that the numbers of putative presynaptic contacts apposed to the GF dendrite are concomitantly increased by En expression. This suggests that En actually promotes the formation of de novo mixed chemical and electrical synaptic contacts. These Brp-sh-labeled contacts probably represent the chemical synapses that have been described using electron microscopy (Sivan-Loukianova and Eberl, 2005), but it will clearly be necessary to confirm that in the future using the same technique.

Interestingly, we were also able to show a redistribution of GF dendritic branches toward the new synaptic inputs. There are 
only a few examples of comparable studies that perturb synaptic specificity in this way. In one, R1-R6 retinal axons were redirected to inappropriate synaptic targets in the medulla by overexpression of the transcription factor Runt (Edwards and Meinertzhagen, 2009), but there was no analysis of the dendritic morphology of the postsynaptic neurons. However, in the olfactory lobe, misexpression of Teneurin guidance molecules in one class of projection neurons (PNs) leads to ectopic synapse formation with incorrect olfactory receptor axons, along with a resultant redistribution of the PN dendrites (Hong et al., 2012).

Our TeTxLC experiments confirmed that fast signaling in the JO-A-GF connection is primarily electrical. This connection has been shown previously to be abolished in shakB mutants and is unaffected by $\mathrm{Cd}^{2+}$ (Lehnert et al., 2013). What, then, is the function of the chemical synapses? JONs and their axons contain choline acetyltransferase (Yasuyama and Salvaterra, 1999) and the GF dendrites express the nicotinic acetylcholine receptor (Fayyazuddin et al., 2006), suggesting that the chemical component of the JON-GF synapse should be excitatory. This argues against the early proposal that it is inhibitory (Strausfeld and Bassemir, 1983), which could have conveniently explained our observation of the reduced response to later SEPs. However, we found that expression of tetanus toxin in the JONs has no effect on this "depression," suggesting that chemical synaptic transmission is not responsible. Other mixed synapses in adult Drosophila also show either limited or no functionality for the chemical component (Trimarchi and Murphey, 1997; Allen and Murphey, 2007). One possibility is that they are required for the development and/or maintenance of the connection; another is that they may release modulatory substances that do not produce acute changes in postsynaptic potential. In any case, the reason for the animal's investment in the construction of these apparently unused chemical synapses remains unknown.

Do the JON-GF electrical synapses rectify? Heterotypic gap junctions between oocytes expressing the isoforms Shaking$\mathrm{B}(\mathrm{Neural}+16)[\operatorname{ShakB}(\mathrm{N}+16)]$ and Shaking-B(Lethal) $[\operatorname{ShakB}(\mathrm{L})]$ are rectifying, that is, they open when the former cell is depolarized relative to the latter and then rapidly close as the $\operatorname{ShakB}(\mathrm{L})$ expressing side of the junction is depolarized (Phelan et al., 2008). Homotypic junctions of either type do not rectify. ShakB $(N+16)$ is expressed by the GF and the giant commissural interneurons (GCIs) to which it is LY-coupled, and ShakB(L) is expressed by TTMn (Phelan et al., 2008). The lack of LY coupling suggests that JONs do not express ShakB(N+16), and it is also unlikely that they express ShakB(L) because the junctions thus formed would rectify in the wrong direction. It is possible that the JON-GF gap junctions are heterotypic and rectifying but formed from a different innexin and ShakB(N+16). Rapid closing of these gap junctions in response to GF depolarization could then be one explanation for the synaptic depression-like phenomenon that we observe. However, there is apparently no indication of such rectification occurring in intracellular current-clamp recordings of JON-GF synaptic potentials with the GF held at different potentials (B. Lehnert, personal communication). In addition, it is not clear why simply increasing the numbers of synaptic connections in response to ectopic En expression would prevent this rectification, although it is possible that the new synapses have a different, nonrectifying, innexin complement.

Partial En and Inv knockdown results in significant decreases in SEP amplitude, suggesting that fewer JONs are firing action potentials and/or that those events are smaller in amplitude. Fewer neurons firing would indicate an effect on the sound transduction mechanisms, whereas smaller action potentials would indicate a partial requirement for En/Inv in promoting neural excitability. The known transduction and amplification components for sound detection are present in all JONs, or at least the A and B types, for example, NompC, Nanchung, and Inactive (Kamikouchi et al., 2009; Sun et al., 2009), so there is no obvious correlation with the expression of En in only a subset of JONs. Relatively few ion channel genes have so far been identified as direct En or Inv binding targets; one exception is Shal, the A-type $\mathrm{K}^{+}$channel (Solano et al., 2003), which could be involved in maintaining excitability during repetitive firing of the JONs, as in larval motoneurons (Ping et al., 2011). Of course, the influence of En and Inv on ion channel expression could be indirect; for example, the gene for Pumilio, which represses the translation of voltage-gated $\mathrm{Na}^{+}$channels (Muraro et al., 2008), is also a putative target of Inv binding (modENCODE Consortium, 2010). Why ectopic expression of En and Inv in JO-B neurons does not conversely increase SEP amplitude is not clear. One possibility is that all of the available sound-responsive JONs are already firing action potentials of maximum possible amplitude; alternatively, the relevant genes may already be activated (or repressed) by other transcription factors in the JO-B neurons.

Partial knockdown of En and Inv also affects the electrophysiology of the JON-GF synaptic connection. Within the limits of our assay, the response to SEP1 is unaffected; however, the response to SEP2 is reduced. To what extent these changes indicate a reduction in the strength of the synaptic connection, rather than a change in its properties, is not yet clear. We were unable to investigate directly whether the numbers of putative synaptic contacts are also reduced, but it certainly appears that enough electrical synapses remain between JO-A axons and the GF to permit NB coupling.

It is likely that, as with the olfactory neuronal precursors in the third antennal segment (Song et al., 2012; Li et al., 2013), the phenotypic identities of the JONs are determined by a combinatorial code of transcription factors, to which En and Inv contribute. A partial reduction of En and Inv probably does not alter the expression of synaptic target recognition molecules enough to prevent synapses from forming with the GF, although it is apparently enough to alter ion channel expression and reduce neuronal excitability. However, the introduction of large amounts of En into a presumably different complement of factors expressed in the JO-B neurons is more disruptive, allowing the expression of cell adhesion molecules that enable the formation of de novo contacts with the GF.

Unlike the olfactory studies, our findings here are the result of the manipulation of En and Inv in postmitotic neurons rather than in their precursors, suggesting a later role for the factors that may be separate from their early function in posterior patterning of the imaginal disk. When we did use a driver (ato-GAL4) that is actually expressed in the precursors but not the early neurons, we found no effect of En knockdown. This apparent postmitotic effect is reminiscent of our previous studies on the cockroach cercal system, which also indicate that En is required postmitotically in sensory neurons to determine first their branching pattern and then their pattern of synaptic connections (Marie et al., 2002). In that system, it seems that, once formed, synapses cannot be altered by En knockdown. Whether this is the case for the JON-GF synapses remains to be determined, because the timing of their development is not known. A detailed study of the timing of their encounter, using GAL4 drivers with different onset times, may shed more light on these processes. 


\section{References}

Allen MJ, Godenschwege TA (2010) Electrophysiological recordings from the Drosophila giant fiber system (GFS). Cold Spring Harb Protoc 2010: pdb.prot5453. CrossRef Medline

Allen MJ, Murphey RK (2007) The chemical component of the mixed GFTTMn synapse in Drosophila melanogaster uses acetylcholine as its neurotransmitter. Eur J Neurosci 26:439-445. CrossRef Medline

Allen MJ, Drummond JA, Moffat KG (1998) Development of the giant fiber neuron of Drosophila melanogaster. J Comp Neurol 397:519-531. CrossRef Medline

Allen MJ, Shan X, Caruccio P, Froggett SJ, Moffat KG, Murphey RK (1999) Targeted expression of truncated glued disrupts giant fiber synapse formation in Drosophila. J Neurosci 19:9374-9384. Medline

Baader SL, Vogel MW, Sanlioglu S, Zhang X, Oberdick J (1999) Selective disruption of "late onset" sagittal banding patterns by ectopic expression of engrailed-2 in cerebellar Purkinje cells. J Neurosci 19:5370-5379. Medline

Bacon JP, Strausfeld NJ (1986) The dipteran "Giant fibre" pathway: neurons and signals. J Comp Physiol A Neuroethol Sens Neural Behav Physiol 158:529-548. CrossRef

Bassemir UK, Strausfeld NJ (1983) Cytology of cobalt-filled neurons in flies: cobalt deposits at presynaptic and postsynaptic sites, mitochondria and the cytoskeleton. J Neurocytol 12:949-970. CrossRef Medline

Blagburn JM (2008) Engrailed expression in subsets of adult Drosophila sensory neurons: an enhancer-trap study. Invert Neurosci 8:133-146. CrossRef Medline

Blagburn JM, Bacon JP (2004) Control of central synaptic specificity in insect sensory neurons. Annu Rev Neurosci 27:29-51. CrossRef Medline

Blagburn JM, Alexopoulos H, Davies JA, Bacon JP (1999) Null mutation in shaking-B eliminates electrical, but not chemical, synapses in the Drosophila giant fiber system: a structural study. J Comp Neurol 404:449458. CrossRef Medline

Boekhoff-Falk G, Eberl DF (2014) The Drosophila auditory system. Wiley Interdiscip Rev Dev Biol 3:179-191. CrossRef Medline

Boerner J, Godenschwege TA (2011) Whole mount preparation of the adult Drosophila ventral nerve cord for giant fiber dye injection. J Vis Exp pii:3080. CrossRef Medline

Booth D, Marie B, Domenici P, Blagburn JM, Bacon JP (2009) Transcriptional control of behavior: engrailed knock-out changes cockroach escape trajectories. J Neurosci 29:7181-7190. CrossRef Medline

Card G, Dickinson MH (2008) Visually mediated motor planning in the escape response of Drosophila. Curr Biol 18:1300-1307. CrossRef Medline

Christiansen F, Zube C, Andlauer TFM, Wichmann C, Fouquet W, Owald D, Mertel S, Leiss F, Tavosanis G, Farca Luna AJ, Fiala A, Sigrist SJ (2011) Presynapses in Kenyon cell dendrites in the mushroom body calyx of Drosophila. J Neurosci 31:9696-9707. CrossRef Medline

Condron BG, Patel NH, Zinn K (1994) Engrailed controls glial/neuronal cell fate decisions at the midline of the central nervous system. Neuron 13:541-554. CrossRef Medline

Dietzl G, Chen D, Schnorrer F, Su KC, Barinova Y, Fellner M, Gasser B, Kinsey K, Oppel S, Scheiblauer S, Couto A, Marra V, Keleman K, Dickson BJ (2007) A genome-wide transgenic RNAi library for conditional gene inactivation in Drosophila. Nature 448:151-156. CrossRef Medline

Duffy JB (2002) GAL4 system in Drosophila: a fly geneticist's Swiss army knife. Genesis 34:1-15. CrossRef Medline

Edwards TN, Meinertzhagen IA (2009) Photoreceptor neurons find new synaptic targets when misdirected by overexpressing runt in Drosophila. J Neurosci 29:828-841. CrossRef Medline

Engel JE, Wu CF (1996) Altered habituation of an identified escape circuit in Drosophila memory mutants. J Neurosci 16:3486-3499. Medline

Fayyazuddin A, Zaheer MA, Hiesinger PR, Bellen HJ (2006) The nicotinic acetylcholine receptor Dalpha7 is required for an escape behavior in Drosophila. PLoS Biol 4:e63. CrossRef Medline

Friedman GC, O'Leary DD (1996) Retroviral misexpression of engrailed genes in the chick optic tectum perturbs the topographic targeting of retinal axons. J Neurosci 16:5498-5509. Medline

Gibert JM (2002) The evolution of engrailed genes after duplication and speciation events. Dev Genes Evol 212:307-318. CrossRef Medline

Guillén I, Mullor JL, Capdevila J, Sánchez-Herrero E, Morata G, Guerrero I (1995) The function of engrailed and the specification of Drosophila wing pattern. Development 121:3447-3456. Medline

Hammer Ø, Harper DAT, Ryan PD (2001) PAST: Paleontological statistics software package for education and data analysis. Palaeontol Electron 4:9pp.

Hammond S, O'Shea M (2007) Escape flight initiation in the fly. J Comp Physiol A Neuroethol Sens Neural Behav Physiol 193:471-476. CrossRef Medline

Homyk T Jr, Szidonya J, Suzuki DT (1980) Behavioral mutants of Drosophila melanogaster. III. Isolation and mapping of mutations by direct visual observations of behavioral phenotypes. Mol Gen Genet 177:553-565. Medline

Hong W, Mosca TJ, Luo L (2012) Teneurins instruct synaptic partner matching in an olfactory map. Nature 484:201-207. CrossRef Medline

Itasaki N, Nakamura H (1996) A role for gradient en expression in positional specification on the optic tectum. Neuron 16:55-62. CrossRef Medline

Jafari S, Alkhori L, Schleiffer A, Brochtrup A, Hummel T, Alenius M (2012) Combinatorial activation and repression by seven transcription factors specify Drosophila odorant receptor expression. PLoS Biol 10:e1001280. CrossRef Medline

Joly W, Mugat B, Maschat F (2007) Engrailed controls the organization of the ventral nerve cord through frazzled regulation. Dev Biol 301:542-554. CrossRef Medline

Kamikouchi A, Shimada T, Ito K (2006) Comprehensive classification of the auditory sensory projections in the brain of the fruit fly Drosophila melanogaster. J Comp Neurol 499:317-356. CrossRef Medline

Kamikouchi A, Inagaki HK, Effertz T, Hendrich O, Fiala A, Göpfert MC, Ito K (2009) The neural basis of Drosophila gravity-sensing and hearing. Nature 458:165-171. CrossRef Medline

Kornberg T (1981) Engrailed: a gene controlling compartment and segment formation in Drosophila. Proc Natl Acad Sci U S A 78:1095-1099. CrossRef Medline

Lehnert BP, Baker AE, Gaudry Q, Chiang AS, Wilson RI (2013) Distinct roles of TRP channels in auditory transduction and amplification in Drosophila. Neuron 77:115-128. CrossRef Medline

Levine JD, Tracey D (1973) Structure and function of the giant motorneuron of Drosophila melanogaster. J Comp Physiol 87:213-235. CrossRef

Li Q, Ha TS, Okuwa S, Wang Y, Wang Q, Millard SS, Smith DP, Volkan PC (2013) Combinatorial rules of precursor specification underlying olfactory neuron diversity. Curr Biol 23:2481-2490. CrossRef Medline

Lienhard MC, Stocker RF (1991) The development of the sensory neuron pattern in the antennal disc of wild-type and mutant (lz3, ssa) Drosophila melanogaster. Development 112:1063-1075. Medline

Logan C, Wizenmann A, Drescher U, Monschau B, Bonhoeffer F, Lumsden A (1996) Rostral optic tectum acquires caudal characteristics following ectopic engrailed expression. Curr Biol 6:1006-1014. CrossRef Medline

Lundell MJ, Chu-LaGraff Q, Doe CQ, Hirsh J (1996) The engrailed and huckebein genes are essential for development of serotonin neurons in the Drosophila CNS. Mol Cell Neurosci 7:46-61. CrossRef Medline

Marie B, Blagburn JM (2003) Differential roles of Engrailed paralogs in determining sensory axon guidance and synaptic target recognition. J Neurosci 23:7854-7862. Medline

Marie B, Bacon JP, Blagburn JM (2000) Double-stranded RNA interference shows that Engrailed controls the synaptic specificity of identified sensory neurons. Curr Biol 10:289-292. CrossRef Medline

Marie B, Cruz-Orengo L, Blagburn JM (2002) Persistent engrailed expression is required to determine sensory axon trajectory, branching, and target choice. J Neurosci 22:832-841. Medline

Marley R, Baines RA (2011) Dissection of third-instar Drosophila larvae for electrophysiological recording from neurons. Cold Spring Harb Protoc 2011.

modENCODE Consortium; Roy S, Ernst J, Kharchenko PV, Kheradpour P, Negre N, Eaton ML, Landolin JM, Bristow CA, Ma L, Lin MF, Washietl S, Arshinoff BI, Ay F, Meyer PE, Robine N, Washington NL, Di Stefano L, Berezikov E, Brown CD, Candeias R, et al. (2010) Identification of functional elements and regulatory circuits by Drosophila modENCODE. Science 330:1787-1797. CrossRef Medline

Morata G, Lawrence PA (1975) Control of compartment development by the engrailed gene in Drosophila. Nature 255:614-617. CrossRef Medline

Morata G, Kornberg T, Lawrence PA (1983) The phenotype of engrailed mutations in the antenna of Drosophila. Dev Biol 99:27-33. CrossRef Medline

Mu L, Ito K, Bacon JP, Strausfeld NJ (2012) Optic glomeruli and their inputs 
in Drosophila share an organizational ground pattern with the antennal lobes. J Neurosci 32:6061-6071. CrossRef Medline

Mu L, Bacon JP, Ito K, Strausfeld NJ (2014) Responses of Drosophila giant descending neurons to visual and mechanical stimuli. J Exp Biol 217: 2121-2129. CrossRef Medline

Muraro NI, Weston AJ, Gerber AP, Luschnig S, Moffat KG, Baines RA (2008) Pumilio binds para mRNA and requires Nanos and Brat to regulate sodium current in Drosophila motoneurons. J Neurosci 28:2099-2109. CrossRef Medline

Orr BO, Borgen MA, Caruccio PM, Murphey RK (2014) Netrin and frazzled regulate presynaptic gap junctions at a Drosophila giant synapse. J Neurosci 34:5416-5430. CrossRef Medline

Patel NH, Martin-Blanco E, Coleman KG, Poole SJ, Ellis MC, Kornberg TB, Goodman CS (1989) Expression of engrailed proteins in arthropods, annelids, and chordates. Cell 58:955-968. CrossRef Medline

Pézier A, Blagburn JM (2013) Auditory responses of engrailed and invectedexpressing Johnston's Organ neurons in Drosophila melanogaster. PLoS One 8:e71419. CrossRef Medline

Pfeiffer BD, Truman JW, Rubin GM (2012) Using translational enhancers to increase transgene expression in Drosophila. Proc Natl Acad Sci U S A 109:6626-6631. CrossRef Medline

Phelan P, Nakagawa M, Wilkin MB, Moffat KG, O'Kane CJ, Davies JA, Bacon JP (1996) Mutations in shaking-B prevent electrical synapse formation in the Drosophila giant fiber system. J Neurosci 16:1101-1113. Medline

Phelan P, Goulding LA, Tam JLY, Allen MJ, Dawber RJ, Davies JA, Bacon JP (2008) Molecular mechanism of rectification at identified electrical synapses in the Drosophila giant fiber system. Curr Biol 18:1955-1960. CrossRef Medline

Ping Y, Waro G, Licursi A, Smith S, Vo-Ba DA, Tsunoda S (2011) Shal/ $\mathrm{K}(\mathrm{v}) 4$ channels are required for maintaining excitability during repetitive firing and normal locomotion in Drosophila. PLoS One 6:e16043. CrossRef Medline

Rørth P (1996) A modular misexpression screen in Drosophila detecting tissue-specific phenotypes. Proc Natl Acad Sci U S A 93:12418-12422. CrossRef Medline

Roy M, Sivan-Loukianova E, Eberl DF (2013) Cell-type-specific roles of $\mathrm{Na}+/ \mathrm{K}+$ ATPase subunits in Drosophila auditory mechanosensation. Proc Natl Acad Sci U S A 110:181-186. CrossRef Medline

Schindelin J, Arganda-Carreras I, Frise E, Kaynig V, Longair M, Pietzsch T, Preibisch S, Rueden C, Saalfeld S, Schmid B, Tinevez JY, White DJ, Hartenstein V, Eliceiri K, Tomancak P, Cardona A (2012) Fiji: an opensource platform for biological-image analysis. Nat Methods 9:676-682. CrossRef Medline

Schneider CA, Rasband WS, Eliceiri KW (2012) NIH Image to ImageJ: 25 years of image analysis. Nat Methods 9:671-675. CrossRef Medline

Sgadò P, Albéri L, Gherbassi D, Galasso SL, Ramakers GMJ, Alavian KN, Smidt MP, Dyck RH, Simon HH (2006) Slow progressive degeneration of nigral dopaminergic neurons in postnatal Engrailed mutant mice. Proc Natl Acad Sci U S A 103:15242-15247. CrossRef Medline

Sharma Y, Cheung U, Larsen EW, Eberl DF (2002) PPTGAL, a convenient Gal4 P-element vector for testing expression of enhancer fragments in Drosophila. Genesis 34:115-118. CrossRef Medline

Shigetani Y, Funahashi JI, Nakamura H (1997) En-2 regulates the expression of the ligands for Eph type tyrosine kinases in chick embryonic tectum. Neurosci Res 27:211-217. CrossRef Medline

Sillitoe RV, Vogel MW, Joyner AL (2010) Engrailed homeobox genes regu- late establishment of the cerebellar afferent circuit map. J Neurosci 30: 10015-10024. CrossRef Medline

Simon HH, Saueressig H, Wurst W, Goulding MD, O'Leary DD (2001) Fate of midbrain dopaminergic neurons controlled by the engrailed genes. J Neurosci 21:3126-3134. Medline

Sivan-Loukianova E, Eberl DF (2005) Synaptic ultrastructure of Drosophila Johnston's organ axon terminals as revealed by an enhancer trap. J Comp Neurol 491:46-55. CrossRef Medline

Solano PJ, Mugat B, Martin D, Girard F, Huibant JM, Ferraz C, Jacq B, Demaille J, Maschat F (2003) Genome-wide identification of in vivo Drosophila Engrailed-binding DNA fragments and related target genes. Development 130:1243-1254. CrossRef Medline

Song E, de Bivort B, Dan C, Kunes S (2012) Determinants of the Drosophila odorant receptor pattern. Dev Cell 22:363-376. CrossRef Medline

Strausfeld NJ, Bassemir UK (1983) Cobalt-coupled neurons of a giant fibre system in Diptera. J Neurocytol 12:971-991. CrossRef Medline

Sun Y, Liu L, Ben-Shahar Y, Jacobs JS, Eberl DF, Welsh MJ (2009) TRPA channels distinguish gravity sensing from hearing in Johnston's organ. Proc Natl Acad Sci U S A 106:13606-13611. CrossRef Medline

Sweeney LB, Couto A, Chou YH, Berdnik D, Dickson BJ, Luo L, Komiyama T (2007) Temporal target restriction of olfactory receptor neurons by Semaphorin-1a/PlexinA-mediated axon-axon interactions. Neuron 53: 185-200. CrossRef Medline

Sweeney ST, Broadie K, Keane J, Niemann H, O’Kane CJ (1995) Targeted expression of tetanus toxin light chain in Drosophila specifically eliminates synaptic transmission and causes behavioral defects. Neuron 14: 341-351. CrossRef Medline

Tanouye MA, Wyman RJ (1980) Motor outputs of giant nerve fiber in Drosophila. J Neurophysiol 44:405-421. Medline

Thomas JB, Wyman RJ (1984) Mutations altering synaptic connectivity between identified neurons in Drosophila. J Neurosci 4:530-538. Medline

Tootoonian S, Coen P, Kawai R, Murthy M (2012) Neural representations of courtship song in the Drosophila brain. J Neurosci 32:787-798. CrossRef Medline

Trimarchi JR, Murphey RK (1997) The shaking-B2 mutation disrupts electrical synapses in a flight circuit in adult Drosophila. J Neurosci 17:47004710. Medline

von Reyn CR, Breads P, Peek MY, Zheng GZ, Williamson WR, Yee AL, Leonardo A, Card GM (2014) A spike-timing mechanism for action selection. Nat Neurosci 17:962-970. CrossRef Medline

Wagh DA, Rasse TM, Asan E, Hofbauer A, Schwenkert I, Dürrbeck H, Buchner S, Dabauvalle MC, Schmidt M, Qin G, Wichmann C, Kittel R, Sigrist SJ, Buchner E (2006) Bruchpilot, a protein with homology to ELKS/ CAST, is required for structural integrity and function of synaptic active zones in Drosophila. Neuron 49:833-844. CrossRef Medline

Wan Y, Otsuna H, Chien CB, Hansen C (2012) FluoRender: an application of $2 \mathrm{D}$ image space methods for $3 \mathrm{D}$ and $4 \mathrm{D}$ confocal microscopy data visualization in neurobiology research. IEEE Pac Vis Symp 2012:201-208.

Wenner P, O’Donovan MJ, Matise MP (2000) Topographical and physiological characterization of interneurons that express Engrailed-1 in the embryonic chick spinal cord. J Neurophysiol 84:2651-2657. Medline

Yasuyama K, Salvaterra PM (1999) Localization of choline acetyltransferaseexpressing neurons in Drosophila nervous system. Microsc Res Tech 45:65-79. CrossRef Medline

Yorozu S, Wong A, Fischer BJ, Dankert H, Kernan MJ, Kamikouchi A, Ito K, Anderson DJ (2009) Distinct sensory representations of wind and nearfield sound in the Drosophila brain. Nature 458:201-205. CrossRef Medline 